\title{
The Trichoderma atroviride putative transcription factor Blu7 controls light responsiveness and tolerance
}

\author{
José E. Cetz-Chel ${ }^{1}$, Edgar Balcázar-López ${ }^{1}$, Edgardo U. Esquivel-Naranjo ${ }^{1,2}$ and Alfredo Herrera-Estrella ${ }^{1 *}$
}

\begin{abstract}
Background: Most living organisms use sunlight as a source of energy and/or information about their environment. Consequently, they have developed mechanisms to sense light quality and quantity. In the fungus Trichoderma atroviride blue-light is perceived through the Blue Light Regulator Complex, which in turn up-regulates a set of genes (b/u) and down-regulates another set $(b / d)$, triggering asexual reproduction. To gain insight into this process, we characterized the blu7 gene, which encodes a protein containing a $\mathrm{C} 2 \mathrm{H} 2$ zinc finger domain.

Results: $\triangle b l u 7$ mutants show reduced conidiation at low light fluences, which is still clear even when exposed to saturating light. For the first time we show a genome wide survey of light regulated gene expression in T. atroviride, including RNA-seq analyses of the wild type and the $\Delta b l u 7$ strains after brief exposure to blue-light. Our data show a reduction in the number of induced genes and an increase in down-regulated genes in the mutant. Light activates stress responses and several metabolic processes in the wild type strain that are no longer activated in the mutant. In agreement with the misregulation of metabolic processes, continuous exposure to white light strongly inhibited growth of the $\triangle b l u 7$ mutant, in a carbon source dependent fashion. RNA-seq analyses under constant white light using glucose as sole carbon source revealed that localization and transport process present the opposite regulation pattern in the $\Delta b / u 7$ and wild type strains. Genes related to amino acid, sugar and general transporters were enriched in the induced genes in the mutant and the repressed genes of the wild type. Peptone supplemented in the media restored growth of the $\Delta b / u 7$ mutant in constant light, suggesting a role of Blu7 in the regulation of nitrogen metabolism in the presence of light.

Conclusions: Blu7 appears to regulate light sensitivity in terms of induction of conidiation, and to play a major role in supporting growth under continuous exposure to light. The diminished conidiation observed in $\Delta b / u 7$ mutants is likely due to misregulation of the CAMP signaling pathway and ROS production, whereas their low tolerance to continuous exposure to light indicates that Blu7 is required for adaptation.
\end{abstract}

Keywords: BLR, RNA-seq, Photoconidiation, Nitrogen metabolism, Glucose, Growth, Light

\section{Background}

Most organisms can perceive light as a signal and the response to this stimulus depends on the length of exposure and light quality, serving as a cue of environmental conditions [1]. Fungi can perceive a wide range of light wavelengths, from far red to ultraviolet (UV) light [2-4]. Many reports on light responses in fungi are

\footnotetext{
* Correspondence: aherrera@langebio.cinvestav.mx

${ }^{1}$ Laboratorio Nacional de Genómica para la Biodiversidad, CINVESTAV Sede Irapuato, Km 9.6 Libramiento Norte Carretera Irapuato-León, 36821 Irapuato, Guanajuato, Mexico

Full list of author information is available at the end of the article
}

related to reproduction, which determines whether they should enter asexual or sexual reproduction or neither [4-8].

The best-characterized photoreceptor in fungi is the White Collar Complex (WCC) of Neurospora crassa. This blue light photoreceptor WCC, formed by the White Collar (WC-1, WC-2) proteins, regulates pigmentation, circadian rhythm, conidiation and phototropism of perithecial beaks [9-11]. The WCC of $N$. crassa controls all light responses characterized so far in this fungus, despite the presence of red (phytochromes), UV (cryptochromes) or green (opsin) photoreceptors in its 
genome [12-15]. However, a cryptochrome dependent oscillator (CDO) driving rhythmic spore development under constant light, where the Frequency/WCC oscillator does not operate, was recently described [16]. The cog-1 (cry-dependent oscillator gate-1) mutation uncovered this CRY (Cryptochrome) dependent oscillator in $N$. crassa, which regulates light responsive genes independently of the WCC. Similarly, as no obvious biological functions for Phy-1/Phy-2 or Nop-1 are reported, their role might be masked by the WCC function. Moreover, in Aspergillus nidulans the phytochrome (FphA) is involved in repression of sexual development and mycotoxin production by red light, whereas the LreA and LreB proteins (orthologues of the WC proteins) stimulate both $[6,17,18]$.

Asexual reproduction induced by light in $T$. atroviride results in the formation of the so called "conidiation ring" in the periphery of the colony. The BLRC, formed by the BLR1 and BLR2 proteins, is responsible for the perception of blue light [19]. Like their $N$. crassa counterparts, the BLR proteins have GATA zinc finger DNA binding domains, that allows them to act as transcription factors, and PAS domains to form a protein complex. The BLRC controls the transcriptional response to light and photoconidiation [19-21]. In T. atroviride a set of genes targeted by the BLRC was identified by microarray analysis after a $30 \mathrm{~min}$ pulse of white light [22]. Rosales et al. [22] reported only 40 differentially expressed genes, 30 blue light up-regulated (blu) and 10 blue light down-regulated (bld). From the 40 light regulated genes, only the blu 7 gene was predicted as a putative transcription factor (TF) encoding a $\mathrm{C} 2 \mathrm{H} 2$ zinc finger DNA binding domain protein. In this sense, microarray analyses covering the complete genome of the fungus $N$. crassa revealed the activation of six genes encoding transcription factors in response to light [15]. However, recently, high-throughput RNA sequencing showed that there are 58 light responsive transcription factors in $N$. crassa [23]. Furthermore, transcriptional regulation by the $N$. crassa's WCC in response to a brief pulse $(8 \mathrm{~min})$ of white light was recently analyzed by Chip-Seq, uncovering more than 400 genes regulated by this complex, including 24 transcription factors (TFs) as direct targets [24]. Currently, only six of the transcription factors, (2 GATA (WC-1, SUB-1), 2 C2H2 (CSP-1, $\mathrm{SAH}-1)$ and $2 \mathrm{Zn}_{2} \mathrm{Cys}_{6}$ (VAD-3 \& Cutinase TF-1ß)) reported by Chen et al., [15] have been studied in response to constant white light exposure. These transcription factors regulate genes after illumination at early or late stages [15]. However, only the absence of the GATA factor sub-1 (submerged protoperithecia-1) showed the expected lack of expression in late light regulated genes after illumination with white light, as a component of a second transcriptional cascade [15]. Despite the lack of late light regulated genes in $\Delta s u b-1$, asexual reproduction and carotenoid accumulation after illumination is similar to that of the wild type (WT); suggesting a main role of sub-1 in sexual reproduction, acting as a repressor of protoperithecia formation [25]. The recent findings by RNA-seq analysis of the light response in $N$. crassa revealed a complex regulation of gene expression during illumination, unveiling down-regulated genes previously not found by microarrary analysis. In addition, TFs negatively regulated after the light treatment were discovered, integrating more pieces to the puzzle of the light response in this fungus [23].

In $A$. nidulans the LreA and LreB proteins regulate 425 genes positively and 108 genes negatively in response to a brief pulse of light, representing $5 \%$ of the genome [26]. Expression of the $f l b C$ gene, a $\mathrm{C} 2 \mathrm{H} 2 \mathrm{TF}$ involved in conidiation, depends on the complex photoreceptor system integrated by the Light Response proteins LreA and LreB as well as the red light photoreceptor FphA. $f l b C$ activation turns on the expression of the transcription factor $b r l A$, a well-known $\mathrm{C} 2 \mathrm{H} 2 \mathrm{TF}$ regulator of conidiation [27]. The photoreceptor complex also activates the expression of $f l b B$ and $f l b D$ encoding bZIP and cMYC TFs, which also regulate the expression of $b r l A$, to promote the morphological transition of vegetative growth to conidiophores in A. nidulans [26]. BrlA regulates the transition of the elongated hyphae to metulae, which in turn activates $a b a A$ expression [28]. AbaA controls the correct formation of conidial beaks, whose maturation is reached upon activation of WetA [29]. T. atroviride has one orthologue of the $\mathrm{flbC}$ gene as putative transcriptional activator but its expression does not appear to be affected by light, similarly to what is observed in A. fumigatus [30]. Despite the fact that there is no obvious $\operatorname{brl} A$ orthologue in the genome of $T$. atroviride, homologues of AbaA (37\% identity with $A$. nidulans) and WetA (A. nidulans, $66 \%$ identity of the C-terminal region) have been identified. Overall, it is clear that there are undiscovered light transcriptional response pathways in T. atroviride. At least part of such pathways must be BLRC targets, such as the putative $\mathrm{C} 2 \mathrm{H} 2$ transcription factor Blu7, acting downstream in response to light. The rapid activation of blu7 expression, by the Blr proteins, showed a maximum at 15 min both under constant illumination and after a pulse of blue light, suggesting a role in the control of early light regulated genes [31].

The cAMP signaling pathway is involved in several processes in fungi, such as growth, reproduction and nutrient utilization [32, 33]. In T. atroviride light stimulates cAMP synthesis and asexual reproduction is stimulated by addition of extracellular cAMP both in the dark and in light on rich medium, but requires the presence of the Blr1 and Blr2 proteins. Intriguingly, the induction 
of asexual sporulation by sudden carbon starvation also requires the presence of the Blr1 and Blr2 proteins, but the addition of extracellular cAMP triggers conidiation even in the absence of the blr1 or blr2 genes [34]. In addition, extracellular cAMP changes the degree of stimulation of conidiation provoked by different carbon sources in the blr mutants of $T$. atroviride, both in a positive and negative way [35]. Furthermore, the carbon source available and the Blr1 or Blr2 proteins act together to stimulate growth and conidiation in the presence of light or in the dark $[35,36]$. These data led to the proposal that the cAMP-signaling pathway regulates conidiation genes through the action of the cAMP dependent kinase (PKA) in coordination with the BLRC in response to light [34]. On top of that, the BLRC dependent PAS domain protein Envoy of Trichoderma reesei, another photoreceptor, regulates cAMP production in the presence of light mainly by inhibition of the corresponding phosphodiesterase, linking regulation of asexual reproduction and nutrient signaling by modulating the expression of the Gproteins Gna1 and Gna3 [37].

The present work describes the role of the putative $\mathrm{C} 2 \mathrm{H} 2$ zinc finger transcription factor Blu7 in the response to light. RNA-seq analysis of the blu7 gene replacement mutants was carried out after a pulse of blue light $\left(100 \mathrm{molm}^{-2}\right)$ to evaluate its role in photoconidiation. The rapid accumulation of the blu7 mRNA in response to light led us to hypothesize that it could be part of a transcriptional cascade resulting in asexual reproduction. The Blr1 and Blr2 dependent induction of asexual reproduction by glucose starvation led us ask if the Blu7 $\mathrm{C} 2 \mathrm{H} 2$ zinc finger protein is involved in this process. Hence, we evaluated the transcriptional response of the $\Delta b l u 7$ mutant when exposed to constant white light on glucose as a carbon source. Surprisingly, the transcriptional analysis of the light response uncovered a role of the blu7 gene in nitrogen regulation in a glucose dependent way.

\section{Results}

\section{Blu7 is commonly found among Hypocreales}

Blue light perception in $T$. atroviride through the Blr1 and Blr2 proteins activates transcription factors to control subsequent events of a transcriptional cascade. One of this putative BLR dependent transcription factors is encoded by the up-regulated gene blu7 [22]. The previously reported blu7 cDNA (642 bp) [22], differed from the gene prediction based on genome sequencing (Id 138208; http://genome.jgi.doe.gov/cgi-bin/dispGeneModel $? \mathrm{db}=$ Triat2\&id $=138208$ ), in that it contained a shorter open reading frame (213 aa). To establish what was the actual gene, we sequenced the CDNA, which corresponded to a 3002 bases long mRNA (GenBank Id KU666056). The CDS corresponded with that of another predicted gene (Id 284873; http://genome.jgi.doe.gov/ cgi-bin/dispGeneModel?db=Triat2\&id=284873; Additional file 1A), and encodes a 537 amino acid protein with a $\mathrm{C} 2 \mathrm{H} 2$ zinc finger DNA binding domain at the C-terminal region, which also contains a Nuclear Localization signal (NLS), two proline rich (ProRich) motifs at the N-terminal region, and a glutamine rich region predicted by MotifScan (http://myhits.isb-sib.ch/cgi-bin/motif_scan) as a putative activation domain [22].

To get insight into the putative function of the Blu7 protein we searched for homologues by BLAST against the non-redundant database (nr) of the NCBI. Orthologues of the Blu7 protein are present in many, but not all ascomycetes, and they are more commonly found in the Hypocreales, being Trichoderma spp., Fusarium, Metarhizium and Colletotrichum spp. the most representative. Interestingly, we did not find orthologues of Blu7 in the $N$. crassa or A. nidulans genomes.

\section{Absence of the blu7 gene results in reduced photoconidiation in $T$. atroviride}

We replaced part of the coding sequence of blu7, covering the polyQ, ProRich, and zinc finger $\mathrm{C} 2 \mathrm{H} 2$ type domain at the $\mathrm{C}$-terminal region of the originally predicted 213 amino acid protein [22], by a cassette containing the selectable marker $h p h$ using the PCR double-joint protocol [38]. Replacement of this locus was validated by PCRs flanking the replacement cassette and confirmed by Southern blot in six independent transformants (Additional file 2A). Although deletion of the blu7 locus was not complete, given that the replacement eliminated the most relevant motifs, we expected to generate a loss of function allele for this putative transcription factor (Additional file 2B).

The growth rate of the mutants was not affected in the dark, neither under constant white light illumination $\left(3.2 \mu \mathrm{molm}^{-2} \mathrm{~s}^{-1}\right)$, as compared to the WT (Additional file 3A). However, a clear growth delay of the three independent $\triangle b l u 7$ mutants tested was observed when the strains were grown under constant blue light (4.9 $\mu \mathrm{molm}^{-2} \mathrm{~s}^{-1}$, Additional file $\left.3 \mathrm{~B}\right)$. Noticeably, during the first $36 \mathrm{~h}$ continuous illumination provoked the strongest effect on growth but by the end of the assay all colonies had the same diameter, suggesting that the mutants do not adapt to or tolerate light normally, and might, upon prolonged exposure to light, be able to compensate for this defect. In contrast, when grown under photoperiods of $12 \mathrm{~h}$ blue light-darkness (4.9 $\mu \mathrm{molm}^{-2} \mathrm{~s}^{-1}$ ) during $72 \mathrm{~h}$, the $\Delta b l u 7$ mutants showed the same growth as the WT (Additional file 3C). We also observed that $90 \%$ more conidia were produced under constant blue $\left(4.9 \mu \mathrm{molm}^{-2} \mathrm{~s}^{-1}\right)$ compared to white light $\left(3.2 \mu \mathrm{molm}^{-2} \mathrm{~s}^{-1}\right)$ in the WT, whereas in the $\Delta b l u 7$ mutants the level of conidiation in blue or white 
light was similar (Additional file 3D). Production of conidia by the $\Delta b l u 7$ mutants under constant white light was $50 \%$ of that observed for the WT, whereas when exposed to blue light conidia production of the mutant dropped to $33 \%$ of that of the WT (Additional file 3D).

Since the BLRC is required to induce the formation of a conidiation ring after a pulse of blue light, and blu7 expression is BLR dependent, we evaluated the production of asexual spores of the mutants when exposed to varying blue light fluence. The conidiation ring was not formed at light fluences lower than $150 \mathrm{mmolm}^{-2}$ in the $\Delta b l u 7$ mutants. Nevertheless, at higher blue light fluences the conidiation ring is observed, but the mutants never reach the yield of conidia of the WT (Fig. 1a, b). Production of conidia is already detectable upon exposure to $50 \mu \mathrm{molm}^{-2}$ of blue light in the WT, whereas the $\Delta b l u 7$ mutants require $150 \mu_{\text {molm }}^{-2}$ to trigger the conidiation process, pointing to either a light perception or downstream signaling defect (Fig. 1a, b). However, the two strains appear to require approximately the same amount of photons to reach half-saturation of the response, suggesting that there is no such a defect in light perception. We then analyzed the expression of the blu7, env1 and, short aerial hyphae 1 (sah1) genes after a pulse of $100 \mathrm{molm}^{-2}$ of blue light by semiquantitative PCR (Fig. 1c). mRNA of blu7 was observed even after 120 min of light induction in the WT and, as expected, it was not detected in the $\Delta b l u 7$ mutant. Expression of the sah1 and env1 genes had the same profile in the WT and the $\Delta b l u 7$ strain after light induction, suggesting that light perception by the BLRC is functional. Thus, the absence of conidiation after exposure to $100 \mu \mathrm{molm}^{-2}$ of blue light is possibly due to the control of a particular set of genes by Blu7.

The transcriptional response to light of the $\Delta b / u 7$ mutant Given that the $\Delta b l u 7$ mutants are unable to form the conidiation ring at $100 \mu_{\text {molm }}^{-2}$ blue light, we decided to analyze the transcriptomes of the WT and a $\Delta b l u 7$ mutant under this condition to identify genes whose a

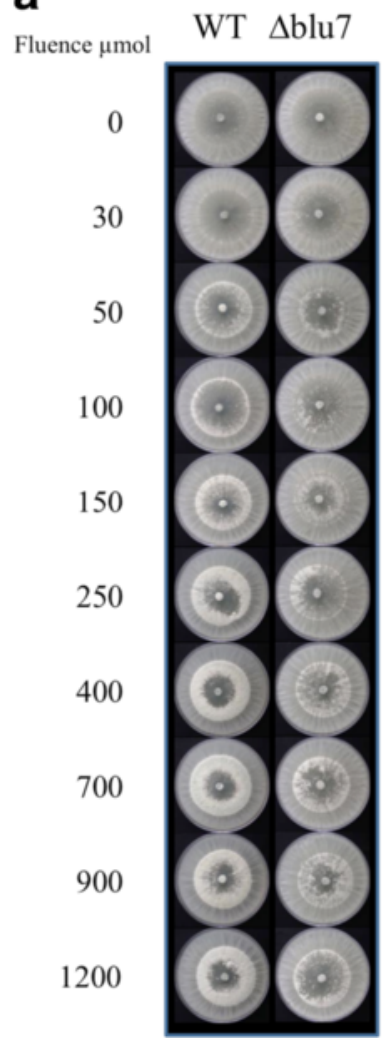

b

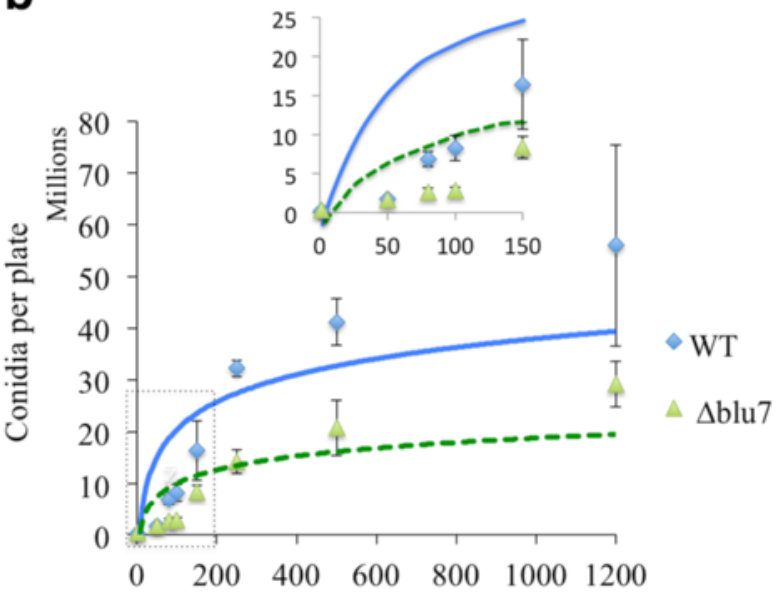

Blue light $\left(\mu \mathrm{mol} / \mathrm{m}^{2} \mathrm{~s}\right)$

C

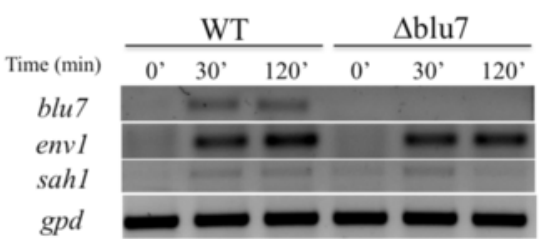

Fig. 1 Photoconidiation of the WT and $\Delta b / u 7$ strains after a pulse of blue light. a Induction of conidiation ring in WT and $\Delta b / u 7$ mutant strains in response to increasing blue light fluence, as indicated. $\mathbf{b}$ Conidia production of the WT (squares) and the $\Delta b / u 7$ (triangles) mutant at different blue light fluence. The inset is a zoom view of the induction of conidiation at fluences lower than $200 \mu \mathrm{molm}{ }^{-2}$. The strains were cultivated during $36 \mathrm{~h}$ in the dark, then exposed to a pulse of blue light of 0,50,80, 100, 250, 500, 1200, 2400, 4800, 9600 and $11000 \mu_{\text {molm }}^{-2}$.c Semiquantitative RT-PCR of the blu7, env1 and sah 1 genes after a pulse of blue light in the WT and the $\Delta b / u 7$ mutant; gpd was used as a control. Replicates of three independent assays were used to evaluate conidia production by the mutant and WT strains. A t-test was applied to data with significant differences set at $P<0.05$. A logarithmic base 10 tendency line was obtained for the data of the WT (blue line) and $\Delta b$ lu7 mutant (green line) 
expression could be affected in the mutant, and that could be involved in asexual reproduction. A total of 453 genes were differentially expressed in the $\Delta b l u 7$ and 461 in the WT in light as compared to controls kept in the dark [cutoff Fold Change $(\mathrm{FC})>2(\log 2|\mathrm{FC}|>1)$, False Discovery Rate (FDR) $<0.01$; Additional file 4]. The WT strain showed more up-regulated genes (246) than the $\Delta b l u 7$ mutant (206), while the $\Delta b l u 7$ mutant presented more repressed genes (247) than the WT (215). The expression levels of the main light responsive genes in the WT and $\Delta b l u 7$ showed similar degree of induction or repression. However, in the WT the light regulated genes in average are more strongly induced (Fig. 2a).

Hierarchical clustering of the differentially expressed genes, based on expression level, results in six main clusters for the total light responsive genes, as compared to their corresponding dark control (Additional file 5A; Fig. 2b). The most responsive genes, up- or downregulated, were grouped in Clusters I and VI (Additional file 4 and Additional file 5A). Cluster II contained induced genes ranging from FC 2.3 to 8 . Cluster III contained genes up-regulated in $\triangle b l u 7$ in a FC range between 2 and 2.5, whereas in the WT ranged from FC 2 to 3.5. In Cluster IV we found the less repressed genes. In Cluster $\mathrm{V}$ the level of repression was similar in both strains, although not in the same genes. Homologues of previously reported light responsive genes in fungi were found distributed in all clusters.

Among the genes regulated in common in both strains we found six genes more strongly induced in $\Delta b l u 7$ (Additional file 6) and 26 genes with higher induction in the WT (Additional file 6). On the other hand, among this set of genes we found 32 more strongly repressed in

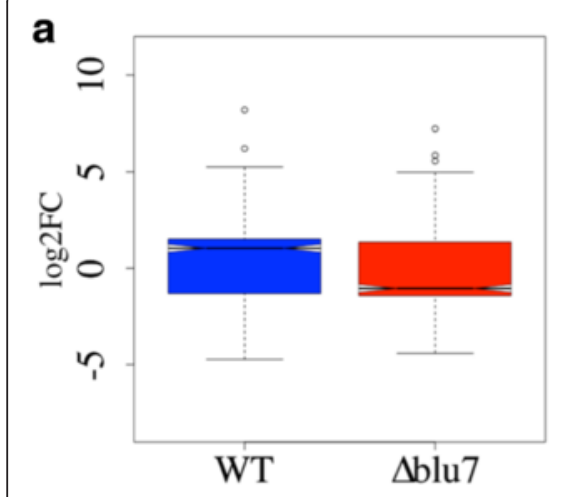

C

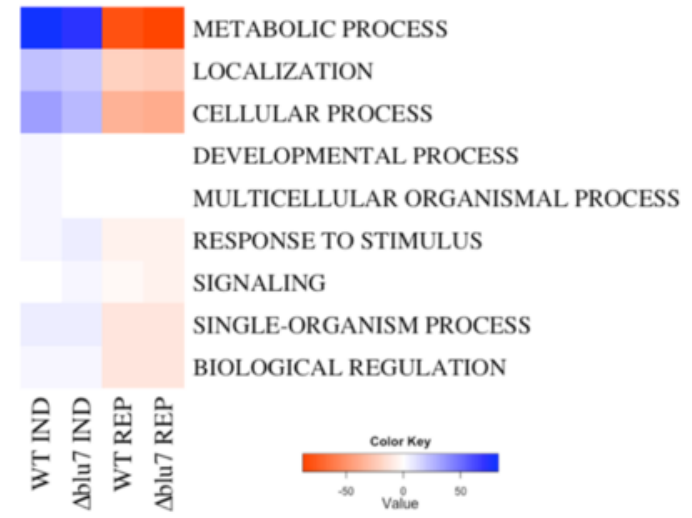

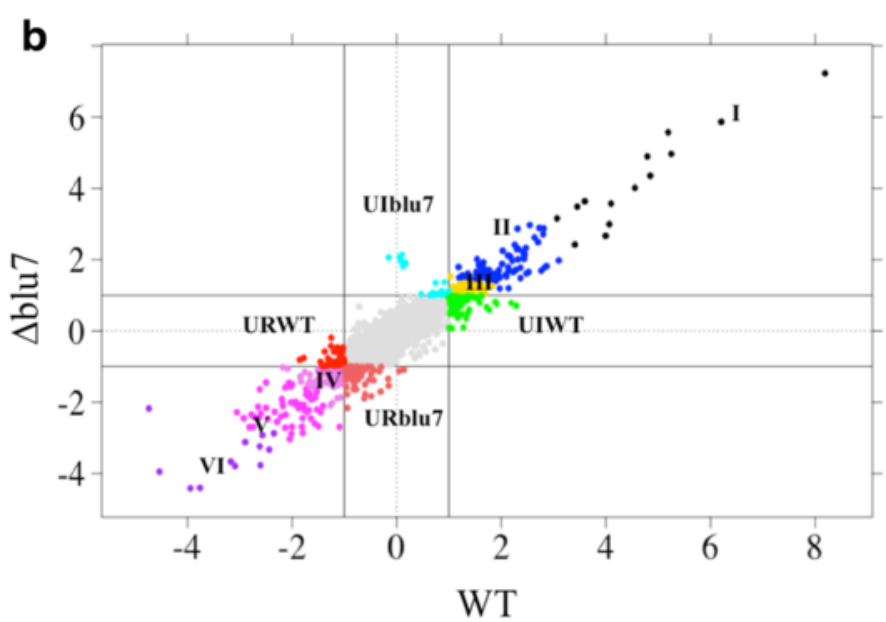

d
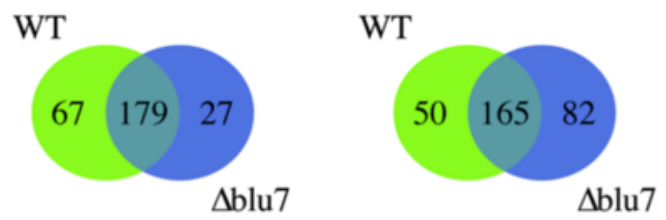

$273 \mathrm{Up}$ regulated genes
297 Down regulated genes

Fig. 2 Transcriptional response of the WT and $\triangle b l u 7$ strains $30 \mathrm{~min}$ after a pulse of blue light. a Boxplot of log2FC of the total differential light regulated genes in the WT and $\Delta b / u 7$ strains, compared to dark control of each strain. $\mathbf{b}$ Plot of the WT vs $\Delta b / u 7$ by log2FC, the resulting 6 clusters of the common regulated genes are indicated with roman numbers. The light induced genes present only in the mutant are localized at the positive $\mathrm{Y}$-axis depicted in cyan and the repressed genes in dark orange in the negative $\mathrm{Y}$-axis. The light induced genes present only in the WT are observed in green at the positive X-axis and the repressed genes are in red at the negative X-axis. c BLAST2GO annotation by biological process of the light regulated genes in the WT or $\Delta b l u 7$ mutant strain. $\mathbf{d}$ Venn diagram showing the overlap in up-regulated (left) and downregulated (right) genes that respond in the WT and $\Delta b / u 7$ mutant strain 
$\Delta$ blu7 (Additional file 7) and 16 genes with stronger repression in the WT (Additional file 7).

In Cluster I we found two alleles (Id. 300570 and Id.: 529689) orthologous to the $N$. crassa ccg-1/grg1 gene (clock controlled gene-1), one of them (Id. 300570) showed the highest level of expression in both the WT $(\mathrm{FC}=292.3)$ and the $\Delta b l u 7(\mathrm{FC}=149.4)$ mutant (Additional file 4). Interestingly env1, a gene related to photoadaptation of light regulated genes, was more strongly induced in the WT $(\mathrm{FC}=73.9)$ than in the $\Delta$ blu $7(\mathrm{FC}=58)$ mutant $(\mathrm{Z}$-score $<0.2)$. The remaining 7 genes in Cluster I were related to catalytic activity, protein binding and nucleotide binding with similar expression profile in the WT and the $\Delta b l u 7$ strain. In Cluster II we found an orthologue of the frq gene of $N$. crassa (Id. 131340), involved in circadian clock regulation, and the blu17 gene (Id. 160158), an orthologue of the $N$. crassa al-3 gene (encoding a geranylgeranyl pyrophosphate synthase). The frq gene had the same level of expression in both strains; but the orthologue of al-3 was slightly more strongly induced in the WT (WT FC=4.2; $\Delta$ blu7 $\mathrm{FC}=2.9$ ). Looking into the transcription factor encoding genes, we detected four in this cluster, three of them corresponding to $\mathrm{Zn}_{2} \mathrm{Cys}_{6}$ zinc finger proteins not yet studied, and the $c p 2$ gene (Id. 319089), an orthologue of the grh-like gene of $N$. crassa involved in the release of spores [39]. Only $c p 2$ showed a slightly stronger induction in the $\Delta b l u 7$ $(\mathrm{FC}=3.8)$ than in the WT $(\mathrm{FC}=3.1)$. Interestingly, the DNA photolyase phrl (Id. 86846) and a putative base excision DNA repair (Id. 26345) protein encoding genes had stronger induction in the WT than in the mutant, both genes related to DNA damage response (Additional file 4).

In Cluster III we detected two transcription factors, the $\mathrm{C} 2 \mathrm{H} 2$ zinc finger protein encoded by azf1 (Id. 165197) and the GATA type sub1 gene (Id. 258818). Induction of the azf1 gene, involved in glucose growth regulation by cyclin control in Saccharomyces cerevisiae $[40,41]$ was stronger in the WT $(\mathrm{FC}=3)$ than in $\Delta b l u 7$ $(\mathrm{FC}=2.1)$. Induction of $s u b 1$ was higher in the $\Delta b l u 7$ mutant $(\mathrm{FC}=2.86)$ than in the WT $(\mathrm{FC}=2)$.

In Cluster IV we found nine genes that were more repressed in the WT than in the mutant, and 13 genes more repressed in the mutant than in the WT (Additional file 4). The genes encoding a calcipresin family protein (Id. 299076), a Ste20 like protein kinase (Id. 242521) were among the most repressed ones in the WT. Calcipresin is a negative regulator of the CalcineurinCalmodulin phosphatase in yeast and mammals [42, 43]. In Aspergillus fumigatus and Botrytis cinerea, calcipresin participates as a positive regulator of hyphal growth [44, 45]. In Cluster V, 6 genes were more repressed in the WT than in the mutant, and 17 genes more repressed in $\Delta b l u 7$ than in the WT. The genes with stronger repression in both strains were detected in Cluster VI (violet, Fig. 2b). The remaining genes were repressed to a similar extent in the $\Delta b l u 7$ and the WT (Additional file 4).

Most of the induced genes in the WT and $\triangle b l u 7$ mutant belong to the GO-term metabolic process (Fig. 2c). We observed the same subcategories of the metabolic process in the WT and $\Delta b l u 7$ strains. However, genes belonging to the lipid metabolic process were more abundant in the WT than in the mutant, whereas in $\Delta b l u 7$ genes belonging to the protein metabolic process were more abundant. The $\Delta b l u 7$ mutant lacked two GO biological process categories (Developmental process and Multicellular organismal process) among the induced genes compared to the WT, whereas the signaling process category was found as induced only in the $\Delta b l u 7$ mutant. In contrast, both the mutant and WT strain down regulated genes showed the same GO categories in biological process, however more genes constituted these categories in the case of the $\Delta b l u 7$ mutant (Fig. 2c). Even though the number of total light regulated genes in each strain is similar, the $\Delta b l u 7$ mutant has less than half of uniquely induced genes (27) of those found for the WT (67), and almost twice the number of unique down-regulated genes (82/50; Fig. 2d).

BLAST2GO annotation by biological process showed that the response to stress, nitrogen metabolism, localization and biosynthetic process were up-regulated only in the WT (Fig. 3a), while processes related to single organism signaling and catabolic process were upregulated only in the $\Delta b l u 7$ mutant (Fig. 3a). Although the same GO biological processes were present in the down-regulated genes of both strains (Fig. 3a), those genes down regulated by light only in the $\Delta b l u 7$ strain showed 12 repressed genes whose expression in the WT remained almost unchanged. Interestingly, more genes repressed only in $\Delta b l u 7$ mutant integrated the GO molecular process hydrolase activity, reported in $T$. reesei as regulated by the BLR proteins and Envoy [46]. The expression profile of the genes 138295 (arrestin binding domain) and 315387 (3-5' cyclic phosphodiesterase), annotated in single organism signaling process, were induced only in the $\Delta b l u 7$ mutant. Those genes annotated as belonging to response to stress, were induced in the WT, and showed low expression levels in the mutant. Aside, genes related to correct protein folding were more strongly repressed in the WT (Fig. 3b). However the $h s p 70$ gene, also related to correct protein folding was induced only in the WT (Fig. 3c). Furthermore, we observed several genes encoding a ribonuclease p-mrp, an Hsp70 protein, a trichotecene c- 15 hydrolase, a glycerol hydrolase, and a peroxisomal catalase with more than two fold induction in the WT that in the mutant did not show a change in expression after the light pulse 
a

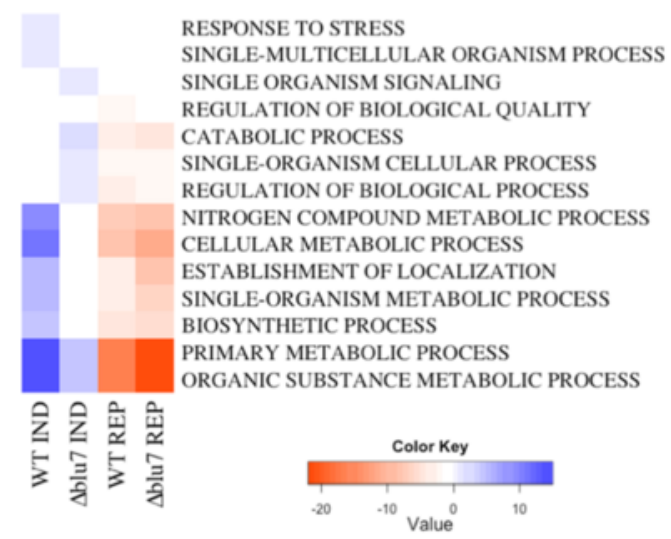

C

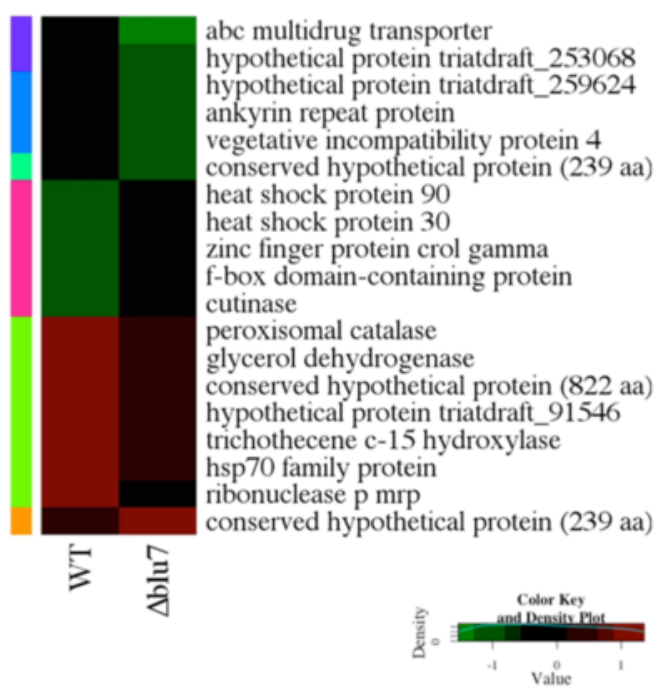

b

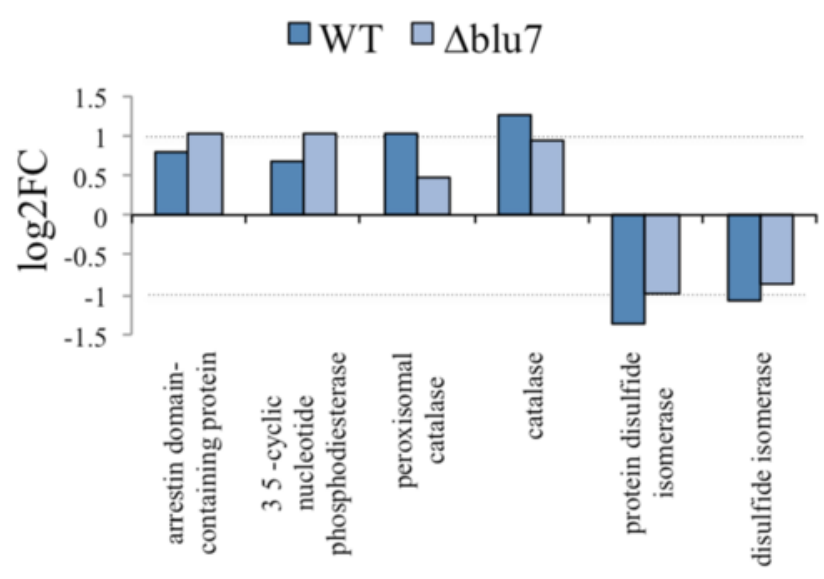

d
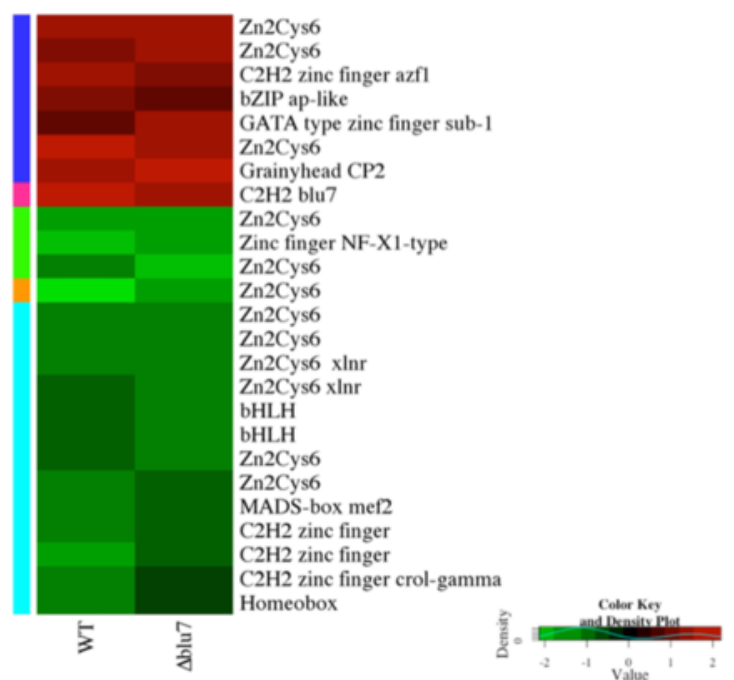

Fig. 3 BLAST2GO annotation of the light regulated genes. a GO annotation by biological processes of the up- or down- regulated genes present only in WT or $\Delta b /$ T. $\mathbf{b}$ Transcriptional profile of selected genes from the transcriptome related to stress and signaling. $\mathbf{c}$ Light regulated genes present only in WT, not responsive in $\Delta b / u 7(1.5>\log 2 \mathrm{FC}>-0.5)$, and light regulated genes present only in $\Delta b / u 7$, not responsive in WT $(1.5>\log 2 \mathrm{FC}>-0.5)$ with hit description by BLAST2GO. $\mathbf{d}$ Heatmap of the transcription factors detected among the light regulated genes of both strains

(Additional file 8), making them potential targets of Blu7 regulation (Fig. 3c).

The molecular function of the most repressed genes $(\mathrm{FC}<0.4)$ in the mutant or the WT strains was related to hydrolase activity, protein binding, transferase activity, and catalytic activity (Additional file 8).

Previous microarray studies in T. atroviride showed light induced expression of only one gene encoding a $\mathrm{C} 2 \mathrm{H} 2$ zinc finger TF (blu7) [22]. Here, we found 25 TFs differentially regulated by light in both strains (Additional file 9). Twelve (48 \%) belong to the $\mathrm{Zn}_{2} \mathrm{Cys}_{6}$ zinc finger family, five (20\%) to the $\mathrm{C} 2 \mathrm{H} 2$ family, including Blu7, and the remaining $32 \%$ to other families (Fig. 3d, Additional file 9). We detected six genes encoding TFs (Ids. 689,
288492, 53602, 173231, 164928, 234627; not studied yet) repressed more than 2 fold only in the WT (Additional file 9). On the other hand, we identified four transcription factor encoding genes repressed more than two fold only in $\Delta b l u 7$.

\section{The Blu7 protein is involved in tolerance to continuous exposure to light}

The reduction of colony growth of the $\Delta b l u 7$ mutant when grown under constant illumination prompted us to evaluate the response of the mutants and the WT under constant blue light. Radial growth and mycelial mass of the WT in darkness and constant exposure to blue light $\left(4.9 \mu \mathrm{molm}^{-2} \mathrm{~s}^{-1}\right)$ was the same on PDA. 
However, a slight radial growth reduction at 10 and 32 $\mu \mathrm{molm}^{-2} \mathrm{~s}^{-1}$ of blue-light was noticed in the WT, although not statistically significant (Additional file 10). In contrast, the $\Delta b l u 7$ mutant strain showed a stronger growth reduction under constant blue light as fluence increased (Additional file 10). The growth delay of the mutant strain was observed already at $4.9 \mu \mathrm{molm}^{-2} \mathrm{~s}^{-1}$ (mycelial mass: $0.150 \pm 0.023 \mathrm{mg}$ in the dark and $0.070 \pm 0.012 \mathrm{mg}$ in light), and was more evident at 10 and $32 \mu \mathrm{molm}^{-2} \mathrm{~s}^{-1}$ of blue light (Fig. 4a). Growth inhibition by light in $T$. atroviride has been reported to be dependent on the BLR proteins and the carbon source available $[35,36]$. Since the BLR complex induces blu 7 gene expression, we evaluated the growth of the mutant under constant white light using glucose, glycerol, mannitol or fructose as sole carbon sources on minimal media and on PDA (Fig. 4b). We observed growth inhibition under constant white light on all carbon sources tested for both the WT and the $\Delta b l u 7$ mutant strains (Fig. 4b). However, the growth reduction of the mutants on medium containing glucose, glycerol or fructose was $40 \%$ more pronounced than that of the WT, whereas the growth rate on mannitol was similar to that of the WT (Fig. 4c). In this regard, it has been clearly established that primary and secondary metabolism processes are regulated by light in fungi $[47,48]$, but how are they regulated is still largely an open question.

To gain deeper understanding of the function of Blu7 in the light response, we obtained an overexpressing strain (OEblu7) of the blu7 gene reported by Rosales et al. [22]. For this purpose the 642 bp CDS was placed under the control of the pyruvate kinase constitutive promoter. The 213 aa long overexpressed version of Blu7 (containing half of the polyQ, the pro-rich and the $\mathrm{C} 2 \mathrm{H} 2$ zinc finger domain) was enough to increase more than twice conidiation after a pulse of blue light compared to WT levels (Additional file 11). Growth of the OEblu7 strain in darkness or in constant light (white or blue) did not differ from that of the WT on minimal media on glucose (Fig. 5a, b). However in constant light colonies of the OEblu7 strain appear to be greener, apparently due to higher production of conidia (Fig. 5a). Indeed, the OEblu7 strain produced $76 \%$ more conidia than the WT under continuous exposure to white light, and $110 \%$ more conidia when exposed to blue light (Fig. 5c), and no inhibition of growth by light was observed (Fig. 5a). The fact that the overexpressing transformant still required light to conidiate indicates that expression of blu7 is not sufficient to trigger conidiation, and that other factors are required to achieve photoconidiation or that the Blu7 protein needs to be posttranslationally modified in a light dependent manner.

Carbon starvation induces asexual reproduction in $T$. atroviride [34]. Interestingly, in the absence of the Blr proteins there is no response to this stimulus [19], suggesting that down stream targets of the Blr proteins regulate conidiation and growth under carbon starvation. The fact that growth of the $\Delta b l u 7$ mutants is reduced when compared to that of the WT, but only in the presence of light, indicates an alteration in their capacity to respond to light rather than a defect on carbon uptake. Consequently, we decided to analyze the transcriptional response of the $\Delta b l u 7$ mutant and the WT strain under constant light using glucose as sole carbon source to identify the genes that are deregulated and involved in growth under such conditions.

\section{Blu7 is involved in glucose metabolism under constant light}

A $\Delta$ blu 7 mutant strain and the WT were cultivated under constant white light or in darkness with $2 \%$ of glucose as sole carbon source during $60 \mathrm{~h}$, and their transcriptome analyzed by RNAseq. Genes showing differential expression under these conditions may include direct targets of BLR, genes regulated by Blu7, and genes regulated by events taking place further downstream in the signaling cascade. Global expression of light regulated genes ranged from 891 to 0.005 FC for the WT, and between 1910 and $0.006 \mathrm{FC}$ for the $\Delta$ blu7 mutant (Fig. 6a). A total of 1901 light regulated genes were identified in both strains (Additional file 12), representing $16 \%$ of the genome. In the WT strain, 1551 light regulated genes were identified and 1380 in $\Delta b l u 7$, as compared to the corresponding controls grown in the dark. Interestingly we found five genes (Ids. 287033, 318140, 274363, 88516 and 267549) induced in WT but repressed in the mutant and two genes (Ids. 91844 and 323077) repressed in the WT but induced in the mutant. Twice as many uniquely induced genes were found in the WT (355) as in the mutant (171), and 633 genes overlapped between the two strains. In contrast, unique down-regulated genes were almost the same number in the WT (173) and the $\Delta b l u 7$ strain (186), and 390 common down-regulated genes were identified (Fig. 6d).

Hierarchical clustering of the light regulated genes results in eight main groups depicted by colors and roman numbers (Additional file 5B, Fig. 6b). In Cluster I (Additional file 12), 13 genes integrated the highly induced genes in both strains, which included the conidiation marker gene con-10, an orthologue of $N$. crassa [49]. Cluster II also has genes highly up-regulated in both strains, however 11 genes were more induced in the WT and 12 were more induced in the $\Delta$ blu 7 mutant (Additional file 12). Interestingly, the env1 gene was more strongly induced in $\Delta b l u 7(\mathrm{FC}=142.3)$ than in the WT $(\mathrm{FC}=57.3)$, suggesting an altered photoadaptation response. Previously reported light responsive genes of fungi were also found in this cluster, such as the 

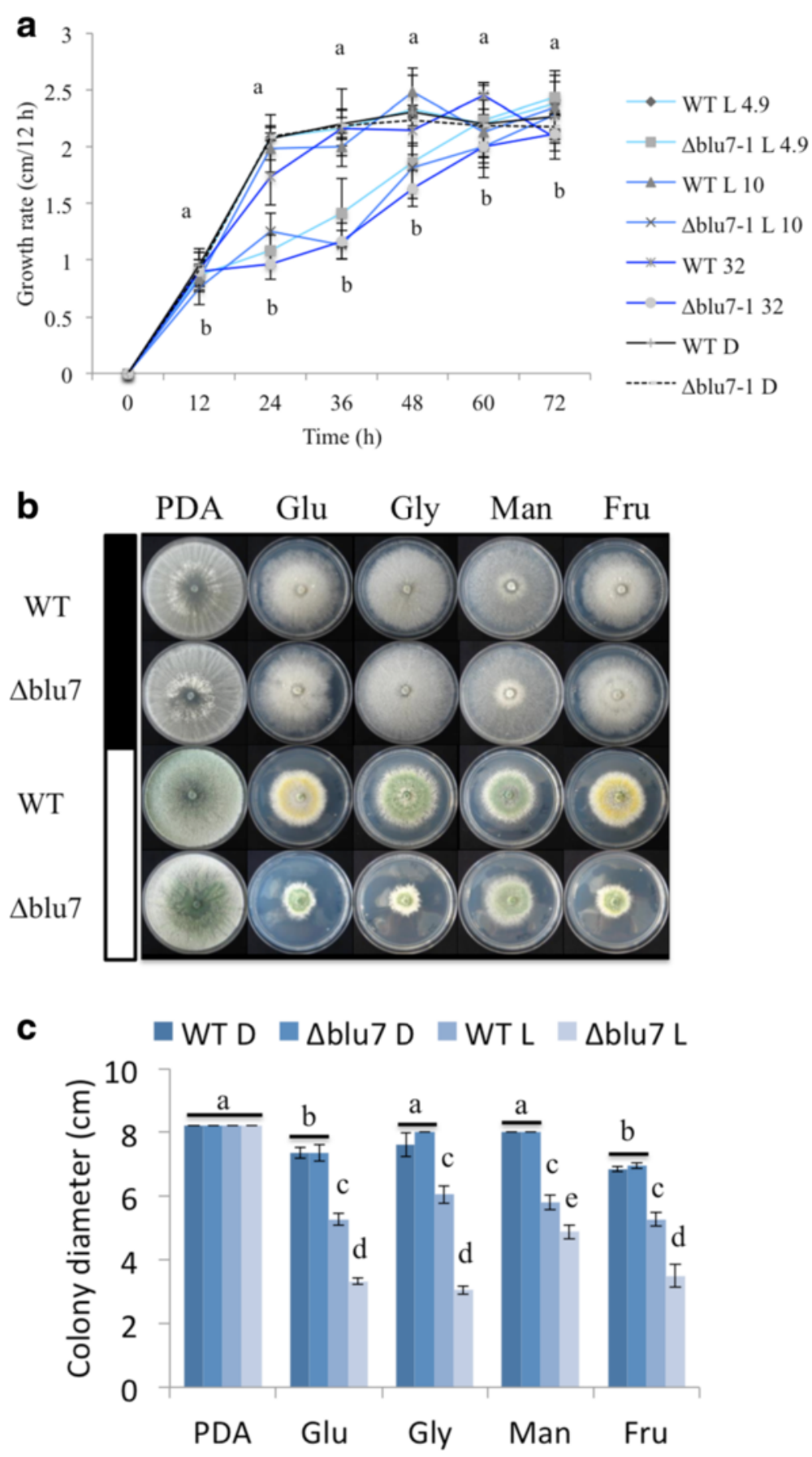

Fig. 4 Reduced growth of the $\Delta b / u 7$ mutant cultivated under constant light. a Colony growth rate of the $\Delta b / u 7$ and WT strains registered every $12 \mathrm{~h}$ during $72 \mathrm{~h}$ when grown under exposure to $4.9,10$ or $32 \mu \mathrm{molm}^{-2} \mathrm{~s}^{-1}$ blue-light on PDA. $\mathbf{b}$ Colonies of the WT and $\Delta b / \mathrm{l} 7 \mathrm{strains}$ grown on minimal media with glucose (Glu), glycerol (Gly), mannitol (Man) or fructose (Fru) as a sole carbon source, PDA was used as control. Colonies were photographed after $72 \mathrm{~h}$ of growth in the carbon source indicated, grown either in the dark (filled bar) or under illumination with white light of $3.2 \mathrm{mmolm}^{-2} \mathrm{~s}^{-1}$ (empty bar). c Colony diameters measured after $72 \mathrm{~h}$ of growth. One-way ANOVA and pairwise $t$-test was applied to the data. Different letters indicate statistically significant differences. Three independent biological replicates were used for each strain

orthologues of the $N$. crassa clock controlled genes 1 and 6 (ccg-1 E ccg-6), and con-13, showing similar induction levels in both strains [48-50]. In Cluster III more genes of the WT showed stronger induction, compared to that found in the $\Delta b l u 7$ strain. GO-terms of those genes indicate that they are involved in metabolism (i.e., cytochrome, oxidoreductase, homoserine o-acetyltransferase), stress responses (i.e., $r d s 1$ and $c r y-D A S H$ ) and transport 


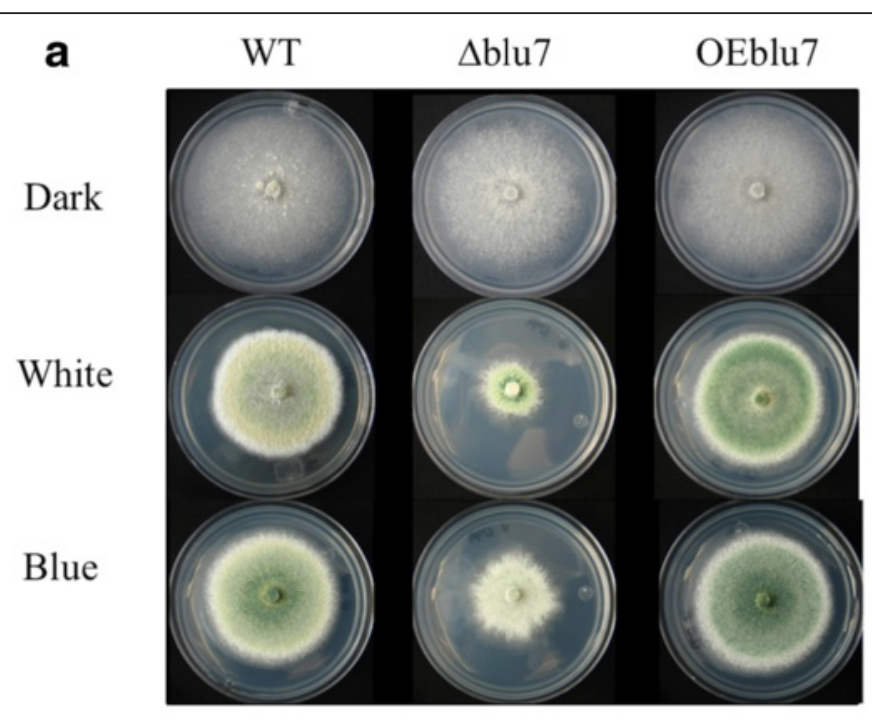

b

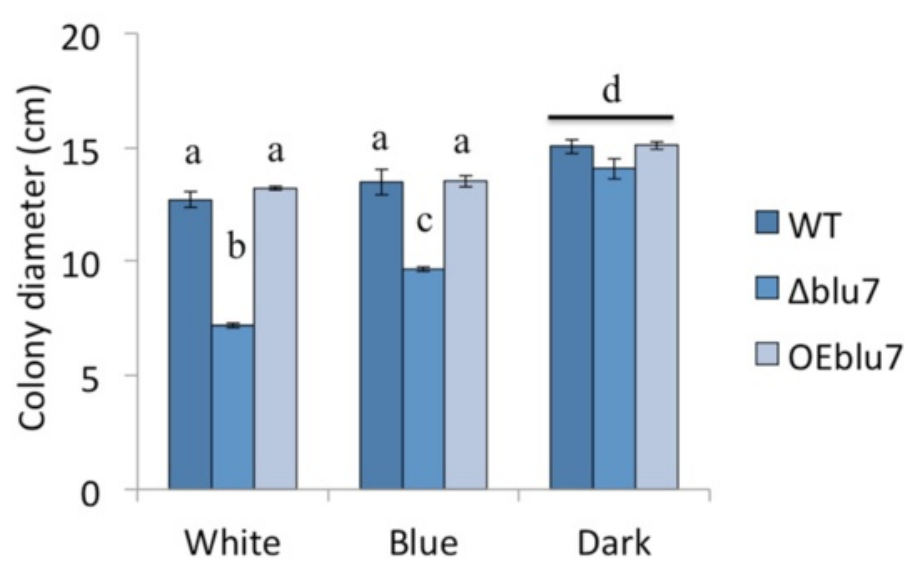

C

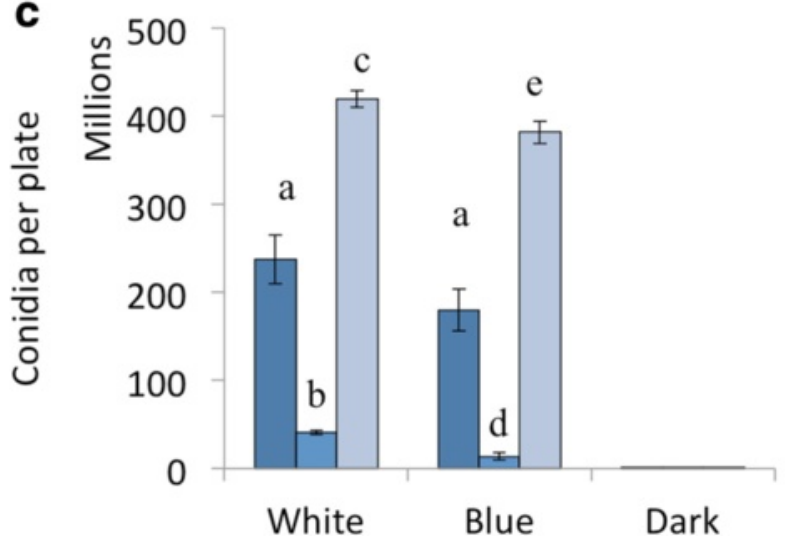

$\square$ WT

$\square$ bblu7

$\square$ OEblu7

Fig. 5 Effect of constant white and blue light effects on conidiation and growth of T. atroviride WT, $\triangle b / u 7$ and OEblu7 strains using glucose as sole carbon source. a Colony growth after $72 \mathrm{~h}$ of growth under constant white $3.2 \mu \mathrm{molm}^{-2} \mathrm{~s}^{-1}$ or blue light $4.9 \mu \mathrm{molm} \mathrm{m}^{-2} \mathrm{~s}^{-1}$ of the $\Delta b / \mathrm{l} 7, \mathrm{WT}$ and OEblu7 strains. b Colony diameter measured after $72 \mathrm{~h}$. c Conidia production of the $\Delta b / \mathrm{l} 7, \mathrm{WT}$ and OEblu7 strains grown under constant white $\left(3.2 \mu \mathrm{molm} \mathrm{m}^{-2} \mathrm{~s}^{-1}\right)$ or blue light $\left(4.9 \mu \mathrm{molm} \mathrm{m}^{-2} \mathrm{~s}^{-1}\right)$. b, c One-way ANOVA and pairwise $t$-test was applied to the data. Different letters indicate statistically significant differences. Three independent biological replicates were used for each strain 


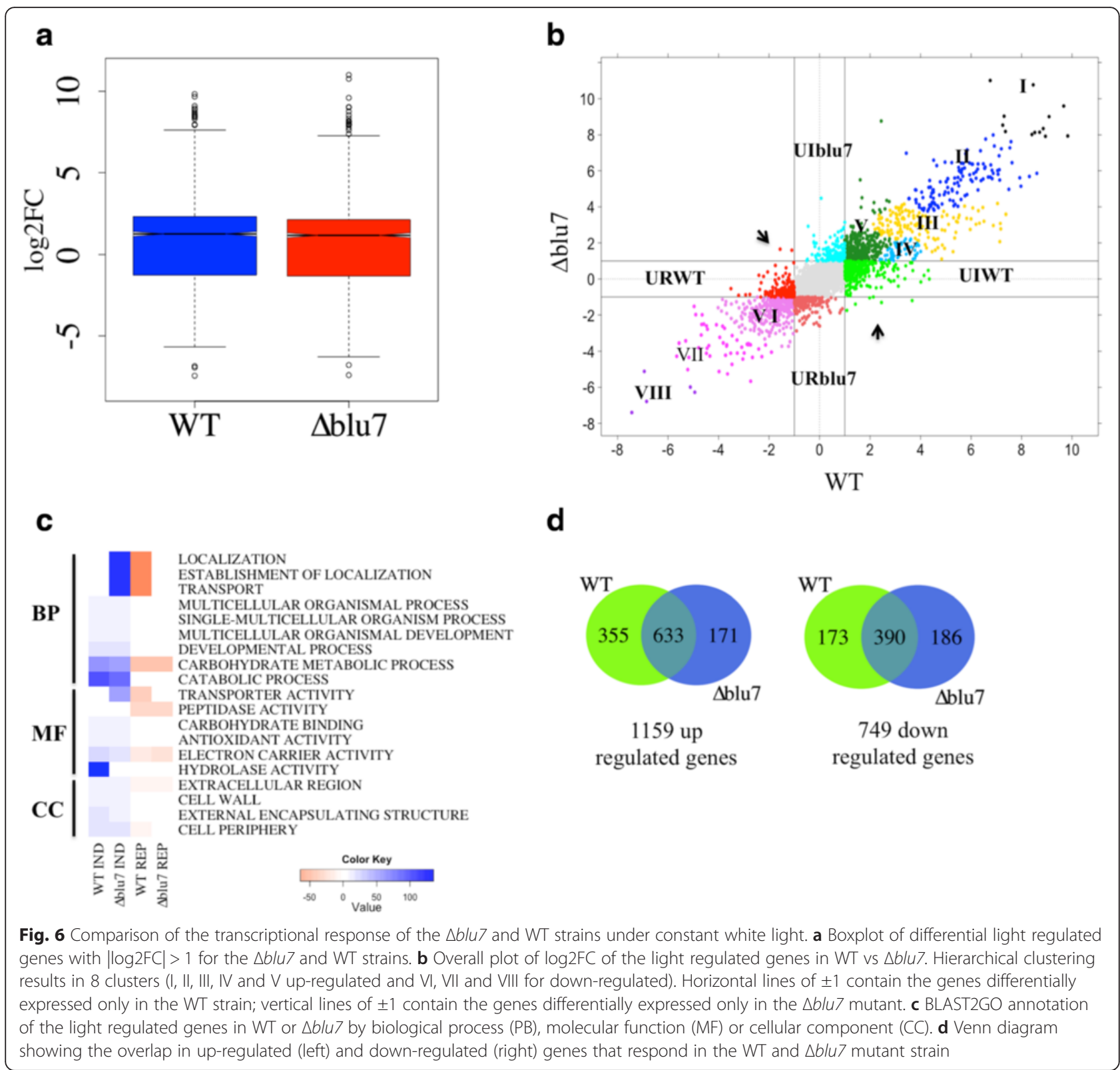

(mfs, multidrug transporter genes). This cluster also encompasses genes that respond more strongly in the $\triangle$ blu7 mutant than in the WT strain, two genes were involved in transport ( $\mathrm{mfs}$ multidrug transporter, general transporter) and 5 genes were annotated in metabolism (aldehyde dehydrogenase, 2og-fe oxygenase family, glyconsiltransferase family, cytochrome, NAD dependent epimerase dehydratase encoding genes). Cluster IV contained genes more strongly induced in the WT than in the $\triangle b l u 7$ mutant (Additional file 11). In this case, the main $\mathrm{GO}$ terms enriched by biological process were related to biosynthetic, metabolic and nucleobase-containing compound metabolic process. In this cluster we found the orthologues of the $N$. crassa clock-controlled gene 9 (ccg-9;
Id. 77441) and regulator of conidiation-1 (Id. 131307) induced to a higher extent in the WT than in $\Delta b l u 7$. Additionally, we observed 14 genes in the WT with more than 3 fold induction compared to the $\Delta b l u 7$ mutant (Additional file 12). Cluster V (Additional file 5B, 11) contains genes with the lowest induction in the WT that in the $\Delta b l u 7$ mutant were more induced. The main genes in this group were annotated in transport and carbohydrate metabolic process. Mainly transporter encoding genes annotated as amino acid permeases and MFS multidrug transporters with more than two fold higher induction in the mutant.

Cluster VI contains slightly repressed genes under continuous illumination $(0.5>\mathrm{FC}>0.06)$. In this case, 
we observed 28 genes repressed more than two fold in $\Delta$ blu7; whereas in the WT strain, 89 of the genes detected showed twice stronger repression compared to the mutant. Genes more strongly repressed in $\Delta b l u 7$ were related mainly to metabolic process, and in the WT to metabolic process and transport. In general, cluster VII contains genes repressed to the same extent in the WT and the $\Delta b l u 7$ mutant. However we detected 16 genes with more than two fold repression in $\Delta b l u 7$, two of them with peptidase activity (a serine endopeptidase and an alpha beta hydrolase) and the remaining genes were related to catalytic activity and hydrolase activity. In the case of the WT, we observed 13 genes repressed more than two fold, as compared to the mutant. In cluster VIII we found the five most repressed genes of the light regulated set. The benzoate 4-monooxygenase cytochrome $\mathrm{P} 450$ encoding gene was more strongly repressed in the WT $(\mathrm{FC}=0.008)$ than in the mutant $(\mathrm{FC}=0.02)$ and the cyclohexanone monooxygenase encoding gene $(\mathrm{FC}=0.012)$ was more strongly repressed in the $\Delta$ blu7 $(\mathrm{FC}=0.012)$ compared to WT $(\mathrm{FC}=0.03)$. While the tricothecene c15-hydroxylase and two cyclohexanone monooxygenase encoding genes were repressed to the same extent in the WT and the $\Delta b l u 7$ strains.

Most of the GO-terms by Biological process (BP), Molecular function (MF) or Cellular component (CC) enriched in the up- or down- regulated genes were present in both strains (Fig. 6c). However, GO-terms enriched of the biological process categories related to Localization, Establishment of localization and transport were induced in the $\Delta b l u 7$ mutant but repressed in WT (Fig. 6c). Interestingly, we observed that the molecular function (MF) related to hydrolase activity (glycoside hydrolase activity family $76,18,15,55,54,81,18$ and 47; as well as chitinases $3,18-1$ and endo-beta glucanases) was enriched only in the case of the WT induced genes. Furthermore, the term transporter activity was enriched in the WT repressed genes, and in the $\Delta b l u 7$ mutant induced ones. These data prompted us to analyze the genes that are light regulated only in one of the strains. Enrichment analysis of the unique up- or downregulated genes in the WT or $\Delta b l u 7$ are shown in Fig. 7a. GO-terms: localization, establishment of localization and transport were enriched in down-regulated genes of the WT and enriched in the up-regulated genes of the $\Delta b l u 7$ mutant. We also observed GO-terms (small molecule binding, nucleoside phosphate binding, phosphotransferase activity, nucleotide binding and protein kinase activity) of induced genes enriched only in WT and GO-terms (transferase activity) of induced genes enriched only in the $\Delta b l u 7$ mutant. On the other hand, down-regulated genes were enriched in the GO-terms carbohydrate metabolic process and catalytic activity in both strains (Fig. 7a). Looking into transport and transporter activity, GO-terms enriched in the genes repressed in the WT and those induced in $\Delta b l u 7$, we observed that these categories were mainly represented by general nitrogen transporter and sugar transporter encoding genes (Fig. 7b). Interestingly, the $\Delta b l u 7$ mutant presented general amino acid transporter and general monosaccharide transporter encoding genes as induced; whereas in the WT methionine transporter and general sugar transporter genes were repressed (Fig. 7b). These data indicate a nitrogen metabolism defect in the mutants, so we cultivated the WT and $\Delta b l u 7$ strains on minimal media supplemented with $0.2 \%$ peptone under constant light during $60 \mathrm{~h}$ (Fig. 8). Radial growth of the WT and $\Delta b l u 7$ mutant was the same under constant light or in darkness when grown on peptonesupplemented media. However, production of conidia in the $\Delta b l u 7$ mutant was much lower than in the WT (Fig. 8), indicating that nitrogen sufficiency is enough to tolerate prolonged light exposure but not to support conidiation in $\Delta b l u 7$ mutants.

\section{Discussion}

Asexual reproduction of $T$. atroviride is induced by light, nutrient limitation and mechanical damage. The BLR complex does not only regulate photoconidiation, but also conidiation in response to sudden carbon deprivation [34]. Notwithstanding, downstream components of the signaling pathway initiated through the BLR proteins are largely unknown. A cDNA microarray analysis identified a single putative transcription factor induced by a pulse of white light. Based on that microarray approach, covering only $12 \%$ of the genes in the genome, 40 genes $(2.8 \%)$ were found to be light regulated. To extend our knowledge of the transcriptional response of $T$. atroviride to light to a genome wide scale, we used RNA-seq to analyze the role of the putative transcription factor Blu7 in the control of asexual development, light sensitivity, and response to light on glucose as carbon source.

The transcriptional response of the WT and $\Delta b l u 7$ mutant, $30 \mathrm{~min}$ after a pulse of $100 \mathrm{molm}^{-2}$ of blue light, showed $4.8 \%$ of the genome responsive with more than 2 -fold change, similarly to $N$. crassa, A. nidulans and $A$. fumigatus $[15,26]$. Interestingly we observed a significant correlation $\left(R^{2}=0.87\right)$ of the up-regulated genes shared between the $\Delta b l u 7$ mutant and the WT, in contrast the down-regulated genes were not correlated $\left(R^{2}=0.57\right)$, showing more negative values in $\log 2 \mathrm{FC}$ of the $\Delta b l u 7$ responsive genes. These data indicate that Blu7 plays a particularly relevant role in tuning the regulation of light repressed genes.

The GO-terms (developmental and multicellular organismal process) induced after the pulse of light only in the WT were related to hypothetical genes, whereas in the signaling category present only in the $\Delta b l u 7$ mutant the genes encoded Envoy1, a 3'-5' cyclic nucleotide 


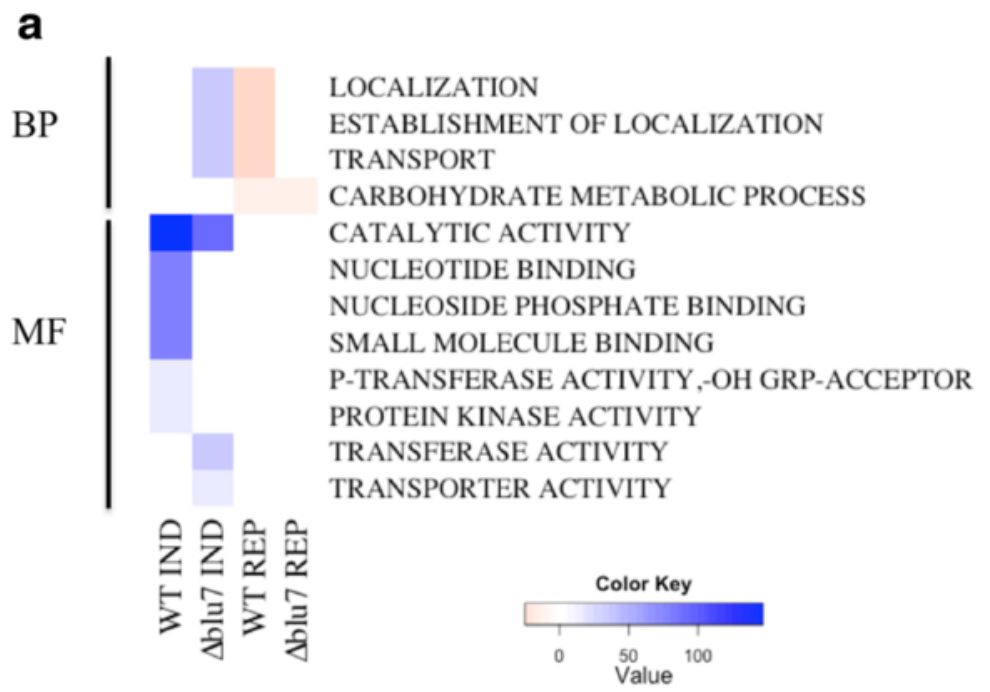

b

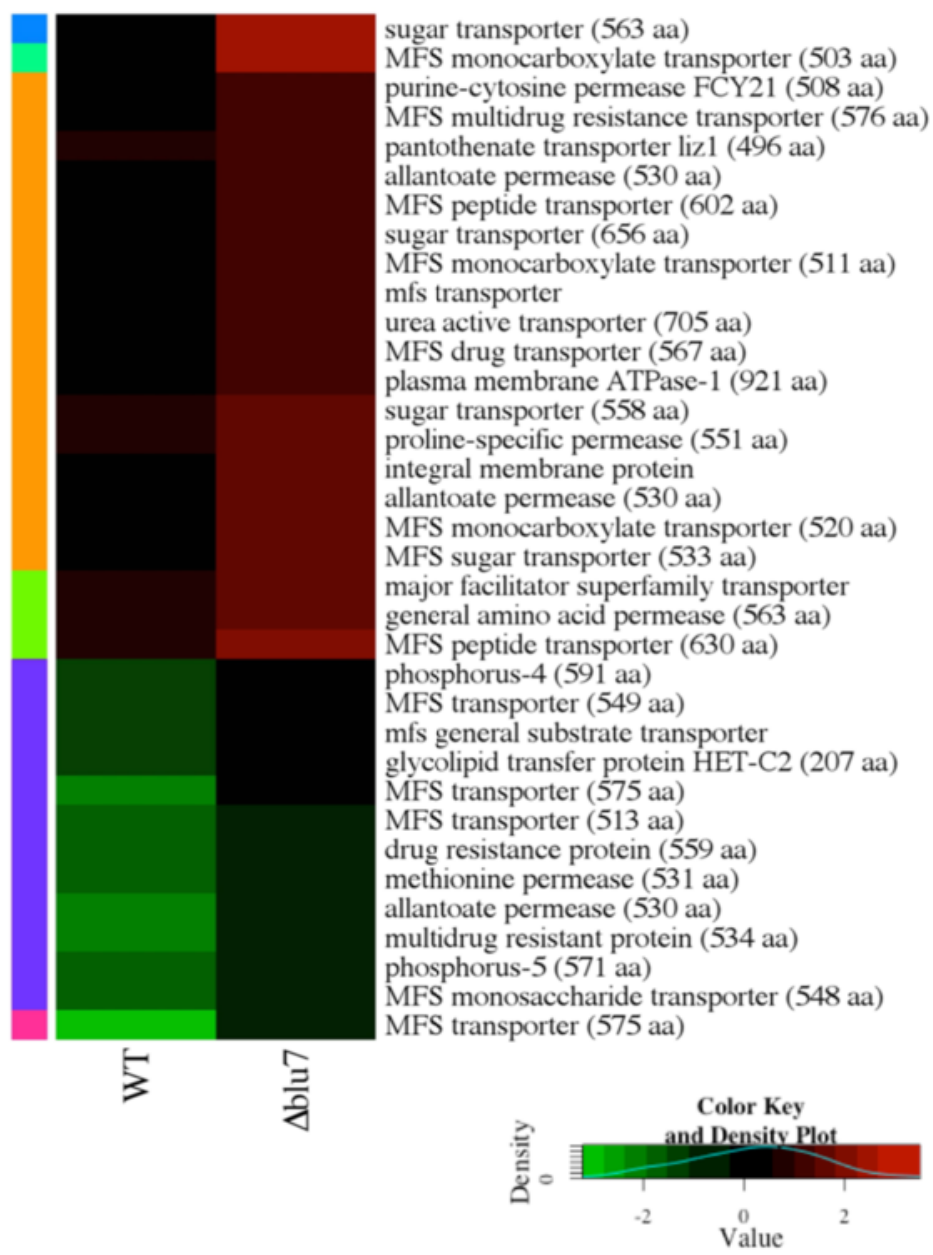

Fig. 7 Analysis of genes differentially expressed only in the WT or the $\Delta b / u 7$ strains. a Enrichment analysis by biological process and molecular function of WT or $\triangle b l u 7$ mutant of unique light regulated genes. $\mathbf{b}$ Heatmap of annotated genes in localization, establishment of localization and transport process in the WT and the $\Delta b / u 7$ strains 


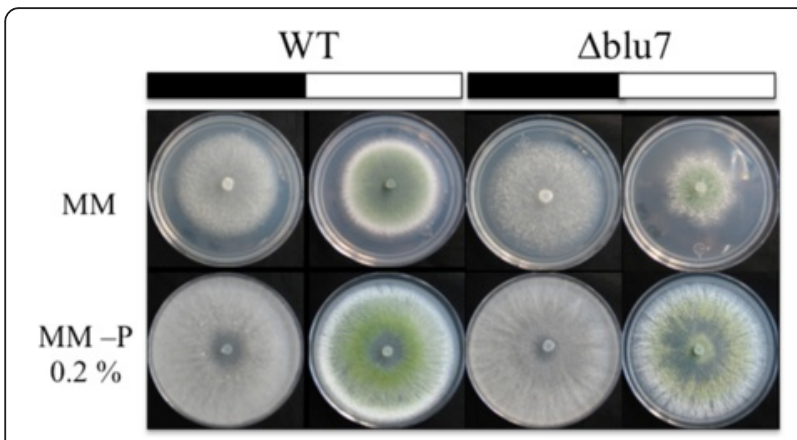

Fig. 8 Growth under continuous exposure to light is rescued by a nitrogen rich medium. Growth of the wild type (WT) and $\Delta b / u 7$ mutant strain under constant white light (filled bars) or in the dark (empty bars), supplemented or not with $0.2 \%$ of peptone, as indicated

phosphodiesterase and an arrestin domain-containing protein. In this regard, it has been established that env1 is an orthologue of the $N$. crassa vivid involved in the negative regulation of light responsive genes in a process called photoadaptation. While the cyclic nucleotide phosphodiesterase controls cAMP levels by hydrolyzing the phosphodiester bond in cAMP. In Trichoderma it is known that there is a transient biphasic oscillation in intracellular cAMP levels, activation of adenylyl cyclase, and phosphorylation of proteins upon exposure to a pulse of blue light. In fact, addition of exogenous cAMP to Trichoderma promotes sporulation even in the dark [51], whereas atropine, a compound known to inhibit adenylyl cyclase in Neurospora [52], prevents sporulation even after photoinduction [53]. In addition, the arrestin gene, which encodes a protein that blocks coupling of the GPCR to G proteins, was more strongly induced in the mutant. Thus, changes in arrestin levels could contribute to lower cAMP production by impairing GPCRs GNA1 or GNA3 signaling. Furthermore, Envoy1 has been postulated as a regulator of cAMP levels in $T$. reesei trough repression of the cAMP phosphodiesterase [37], and the G-protein coupled receptors GNA1 and GNA3 direct that control of cAMP levels by regulating env1 and their own expression. In agreement with our observations of the light induction of blu7, the corresponding mutants are less responsive to light, hence to conidiation, possibly due to reduced phosphodiesterase repression and partial loss of cAMP signaling of GNA1 or GNA3 by the presence of the Arrestin protein. Although, the cAMP accumulation in the $\triangle b l u 7$ mutant after the pulse of blue light requires further investigation in order to validate this hypothesis. Taking together these data, we suggest that in the T. atroviride WT strain cAMP might allow asexual reproduction after the pulse of light, but in the $\Delta b l u 7$ strain the missing signaling impairs conidiation at low light fluence. At high blue light fluence another level of regulation may exist, such as the accumulation of ROS or DNA damage, to induce conidiation in order to survive to extreme environments.

In agreement with these observations, a peroxisomal catalase and one heat shock protein were induced only in the WT, and another catalase was more strongly induced in the mutant than in the WT. Thus, the typical activation of stress responses observed in several fungi after exposure to light [54] is reduced in the Trichoderma $\Delta b l u 7$ mutants. It has been observed that oxidative stress stimulates differentiation in $N$. crassa, Aspergillus and Trichoderma [36, 55-57], and ROS produced upon light exposure induces conidiation in $N$. crassa [58]. In this sense, catalases and superoxide dismutases reduce ROS after the light pulse to re-establish cell homeostasis. Furthermore, it was recently shown that in B. cinerea the white collar proteins are required to deal with ROS produced under constant illumination, and that the Bcltf1 GATA TF, a homologue of SUB-1 of N. crassa, is important to cope with oxidative stress [59]. In the $\Delta b l u 7$ mutant described here we observed a higher expression of $s u b-1$, which might, consequently result in lower levels of ROS after exposure to light, and might play a particularly relevant role at low light fluences.

Another possible explanation for the reduced catalases transcript levels and lower conidiation levels in the $\Delta b l u 7$ mutants is that there is a higher reducing power in these mutants than in the WT. In this regard, we observed 11 genes with oxidoreductase activity repressed only in the mutant. Also, we observed 6 genes encoding enzymes with reductase activity (cyclohexanone monooxygenase, lipooxigenase 1 , berberin family protein, FAD monooxygenase, FAD binding domain protein, NADPH dehydrogenase) more strongly induced in $\Delta b l u 7$ and 4 genes (encoding: short chain dehydrogenase reductase, oxidoreductase protein, FAD dependent oxidoreductase, $\mathrm{NADH}$-flavin oxidoreductase NADH oxidase family protein) repressed only in the WT. In addition, there were 2 genes (encoding cyclohexanone monooxygenase, \& FAD monooxygenase) repressed to a lower extent in the $\Delta b l u 7$ mutant (FC $0.49,0.47)$ than in the WT strain (FC 0.36, 0.36). Therefore, the reduced levels of ROS signaling and cAMP production would not be sufficient to trigger conidiation in the $\Delta b l u 7$ mutants at low light fluence. This defect might be compensated at high light fluence by higher production of ROS, although under those conditions these signals might be regulated through another mechanism.

In $N$. crassa and A. nidulans the WCC controls several transcription factors that in turn regulate asexual reproduction $[15,17,26,60]$. Among the transcription factors regulated by a pulse of blue light only 6 have GO annotation. We observed two TF (cp2 and sub-1) more strongly induced in the $\Delta b l u 7$ mutant. GRHY-like or CP2 has been found in several 
fungal transcriptomes of light treatments $[15,24,26]$. Recently a CP2/GRHY-like was identified as a conidial separation-2 (csp-2) allele in $N$. crassa and shown to be involved in conidial separation and spore release by cell wall remodeling [39]. Reports on the study of a sub-1 deletion mutant showed that its presence is required to activate late light regulated genes in $N$. crassa. However, the mutant presents defects only in sexual development $[15,25]$. It might be that some of the genes more strongly induced in the $\Delta b l u 7$ mutant are not directly under its control, but at least are in part regulated by CP2 or SUB-1.

Under constant illumination, we observed a growth delay in the $\Delta b l u 7$ mutant at high light intensities (above $\left.10 \mu \mathrm{molm}^{-2} \mathrm{~s}^{-1}\right)$. This is in agreement with the requirement of the BLR proteins to grow in the presence of blue or red light in T. atroviride [19]. Under constant illumination the BLRC must regulate genes to deal with the constant exposure to light (i.e., growth, morphogenesis, stress). The growth delay of the mutants under constant blue light suggests that Blu7 participates in light tolerance. Reduced tolerance of the $\Delta b l u 7$ mutants was much more evident when glucose was used as sole carbon source compared to rich media, such as PDA, suggesting a role of Blu7 in carbon metabolism to tolerate continuous light exposure. The combination of light with different carbon sources differentially stimulates conidiation and growth, and this response depends on the BLR proteins $[35,36]$. In addition, the downstream target of the BLRC, Envoy1 of T. reesei is involved in carbon dependent growth in the presence of light.

Since light only temporarily delayed growth of the mutants, we suggest that they are affected in their capacity to tolerate light. These phenotypes resemble those of the $\Delta e n v 1$ mutants of $T$. reesei and T. atroviride in constant light on PDA [61, 62]. In the env1 mutants of $T$. reesei the lack of the negative feedback loop, over the light regulated genes controlled by the BLRC, leads to a reduced growth [61, 62]. In this loop Envoy acts as a repressor of negative regulators of growth activated by the BLR proteins. Accordingly, the stronger induction of env1 in $\Delta b l u 7$ suggests that Blu7 participates as a positive regulator of growth in this negative feedback loop; however, further investigation is required to test this hypothesis.

Conidiation of the $\Delta b l u 7$ mutant was not completely impaired but reduced in constant light. This is also in agreement with the phenotype of $\Delta e n v 1$ mutants of $T$. atroviride which produce more conidia than the WT strain in response to light, likely due to a longer permanence of mRNAs of photoconidiation genes, such as blu7 [62]. In the $\Delta b l u 7$ mutant, the increased induction of env1 may lead to fast shut down of the photoconidiation genes and the absence of Blu7 in reduced cAMP levels, due to increased transcript levels of the cAMP phosphodiesterase gene, and thereof of its enzymatic activity.

The differentially expressed genes observed in the $\Delta b l u 7$ and the WT grown on glucose containing medium under continuous exposure to white light reflected major metabolic changes in both strains compared to darkness. Since in darkness, growth of the WT and the $\Delta b l u 7$ mutant was similar, glucose uptake or metabolism is not deficient or impaired. This points to a light activation of the Blu7 protein or its light regulation through an interacting protein to support metabolic changes. Interestingly, GOterms related to localization and transport categories were repressed in the WT and induced in the $\Delta b l u 7$ mutant. These categories contained genes encoding sugar, amino acid and multidrug transporters. In addition, we observed two-fold higher induction of the nmrA gene (Id $35890)$ in the WT $(\mathrm{FC}=3.9)$ compared to the $\Delta b l u 7$ mutant $(\mathrm{FC}=1.8)$. In $A$. nidulans, $\mathrm{NmrA}$ is a negative regulator of nitrogen metabolite repression, which controls expression of enzymes and permeases necessary for the use of non-preferred nitrogen sources [63, 64]. Thus, the partial nitrogen metabolism deregulation observed in the $\Delta b l u 7$ mutant might be due to $n m r A$ repression, leading to the induction of several amino acid transporter genes in $\Delta b l u 7$. In $M$. oryzae the tps 1 gene (encoding a trehalose-6-phosphate synthase), a glucose-6-phosphate sensor, negatively regulates NMR inactivating Nmr1-3. Also the multidrug and toxin extrusion (MATE)-family pump Mdt1, a downstream target of Tps1, is involved in glucose assimilation, conidiation and virulence [65]. Similarly, we observed several monosaccharide and general sugar transporters induced in $\Delta b l u 7$ and repressed in the WT, as well as multidrug transporters induced in both strains. These observations point to the light regulation of carbon and nitrogen metabolism to stimulate conidiation and growth in $T$. atroviride, respectively.

Carbon or nitrogen starvation results in the formation of less branched compact hyphae than those observed in rich media, in addition the sudden lack of nitrogen or carbon source in $T$. atroviride resembles the ring of conidiation triggered by a pulse of light [19]. Interestingly, in the absence of the blr1 or blr2 genes the induction of conidiation by carbon (glucose) starvation is lost, in contrast nitrogen starvation can still stimulate conidiation, suggesting an independent regulation of asexual reproduction by the BLR proteins [34]. Thus we hypothesized that nitrogen metabolism should be involved in hyphal growth under constant light. Several genes encoding enzymes with peptidase activity (papain cysteine protease, candidapesin-3 precursor, family a1 protease, microbial serine protease, extracellular alkaline serine protease), including an intracellular serine protease, were induced in the WT strain, suggesting that protein 
degradation or recycling of nitrogen resources is activated in response to light. Nitrogen metabolism related genes have also been found among the light regulated genes of $N$. crassa $[15,23]$.

In accordance with these observations, we found an allantoinase-encoding gene (Id. 300514), involved in purine metabolism, induced only in the WT. Besides two allantoate permeases (Ids. 257840 \& 127784) were repressed in the WT, but not in the $\Delta b l u 7$ strain, and two allantoate transporters (Ids. 302043 \& 35866) were induced only in the mutant - allantoate is used as nitrogen storage in plants [66]. Transport of amino acids was repressed in the WT, mainly methionine transport. In contrast, in $\Delta b l u 7$ higher induction levels of genes encoding amino acid transporters and allantoate transporters were observed. These data are highly coincident with the transcriptional response to nitrogen starvation reported in the phytopathogenic fungus Magnaporthe grisea [67]. Taken together, our data appear to indicate that the absence of Blu7 mimics a nitrogen starvation condition when Trichoderma is grown under constant exposure to light, which results in clearly reduced growth. Restoration of growth rate and radial colony size of the WT and $\Delta b l u 7$ mutants by the addition of peptone to the media suggests that light growth inhibition is mainly due to nitrogen intracellular metabolism, and that Blu7 indirectly participates in the regulation of nitrogen metabolism in the presence of light under limited nitrogen supply (Fig. 8). As a consequence of the lack of Blu7, the mutants have lower capacity to recycle nitrogen sources, requiring the induction of general amino acid transporters, which in the presence of peptides from peptone are taken up and metabolized, resulting in much better growth.

We also observed misregulation of the ras GTPase (rsr1; Id. 300901), which was induced to higher levels in the $\Delta b l u 7(\mathrm{FC}=5.2)$ mutant than in the WT $(\mathrm{FC}=2.0)$. Activation of this pathway in yeast involves a G-protein coupled receptor and RAS signaling to regulate glucose availability $[68,69]$. From the GPCRs regulated in both strains only the predicted GPCR gene Id. 40423 is more strongly induced in the $\triangle b l u 7$ mutant, which might act as an upstream regulator. GPCRs are involved in amino acid and carbon source sensing as well as in cAMP perception. The gpr1 gene (Id. 83166), encoding a homolog of the N. crassa cAMP receptor Gpr1 [70, 71], is induced only in the WT strain. Silencing of gprl in T. atroviride $\mathrm{P} 1$ reduces growth, conidiation and secondary metabolism [71]. In Yeast the GPR1-GPA2 GPCR system is involved in the regulation of cAMP signaling [69]. The absence of Gpr1 in the $\Delta b l u 7$ mutants in conjunction with the increase in cAMP phosphodiesterase might be reflected in the lack of protein kinase A (PKA) activity resulting in the loss of this signaling pathway to regulate the light response. PKA signaling after a pulse of light is required to regulate up or down responsive genes, also regulated by the BLR proteins $[19,34]$. We further observed 6 serine-threonine protein kinase encoding genes induced only in the WT, which might exert posttranslational modifications in transcription factors, consequently affecting regulation of gene expression in response to light, or might directly affect enzyme activity, resulting in altered metabolism.

An overview of the data presented here is depicted in Fig. 9. The BLRC perceives light and induces blu7 expression. Transient increase of cAMP by inhibition of the 3'-5' cAMP phosphodiesterase leads to activation of photoconidiation genes by PKA. Growth rate under constant light depends on nitrogen availability, controlling the uptake from the media or mobilization of stored nitrogen (Fig. 9).

\section{Conclusions}

The present work describes the transcriptional response to light of the mutant in the putative transcription factor encoding gene blu7, a BLRC dependent light regulated gene in T. atroviride. The blu7 gene is required for photoconidiation at low but not at high blue light fluence. The absence of blu7 resulted in increased levels of expression of the 3'-5' cAMP phosphodiesterase encoding gene, which could explain the reduced conidiation observed in the mutant. Furthermore, the increased expression of env1 observed in the $\Delta b l u 7$ strain under constant illumination suggests linked roles in photoadaptation between these two regulators. Interestingly, the growth inhibition response of the blu7 replacement mutant to light was reduced in rich media. The decreased expression of energy and metabolism related genes in the $\triangle b l u 7$ mutant under constant light may explain the slower growth, rendered by defective nitrogen metabolism.

\section{Ethics (and consent to participate) \\ Not applicable.}

\section{Consent to publish}

Not applicable.

\section{Methods}

\section{Strains and culture media}

All Trichoderma strains were cultivated on potato dextrose agar (PDA) at $28{ }^{\circ} \mathrm{C}$ under the light regime indicated. The wild type strain used was IMI206040. The $\Delta$ blu7 mutant was obtained by replacement of $664 \mathrm{bp}$ of the gene locus of the WT strain IMI206040. An overexpressing version of blu7 was generated using the $p k i$ (pyruvate kinase) constitutive promoter inserted in plasmid pUE10 [31]. Culture minimal medium (MM) contains $\mathrm{K}_{2} \mathrm{HPO}_{4} 5.1 \mathrm{mM}, \mathrm{MgSO}_{4} 1.7 \mathrm{mM}, \mathrm{KCl} 2.7 \mathrm{mM}, \mathrm{NH}_{4} \mathrm{NO}_{3}$ 


\section{a Pulse of blue light}

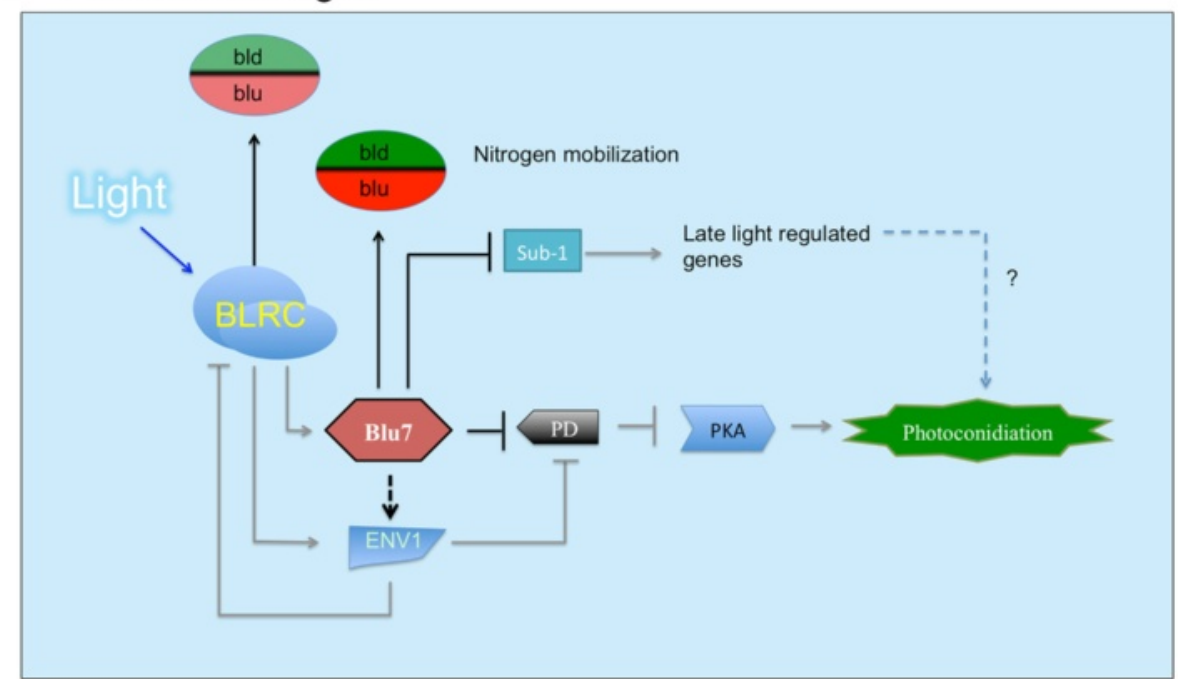

b Constant white light

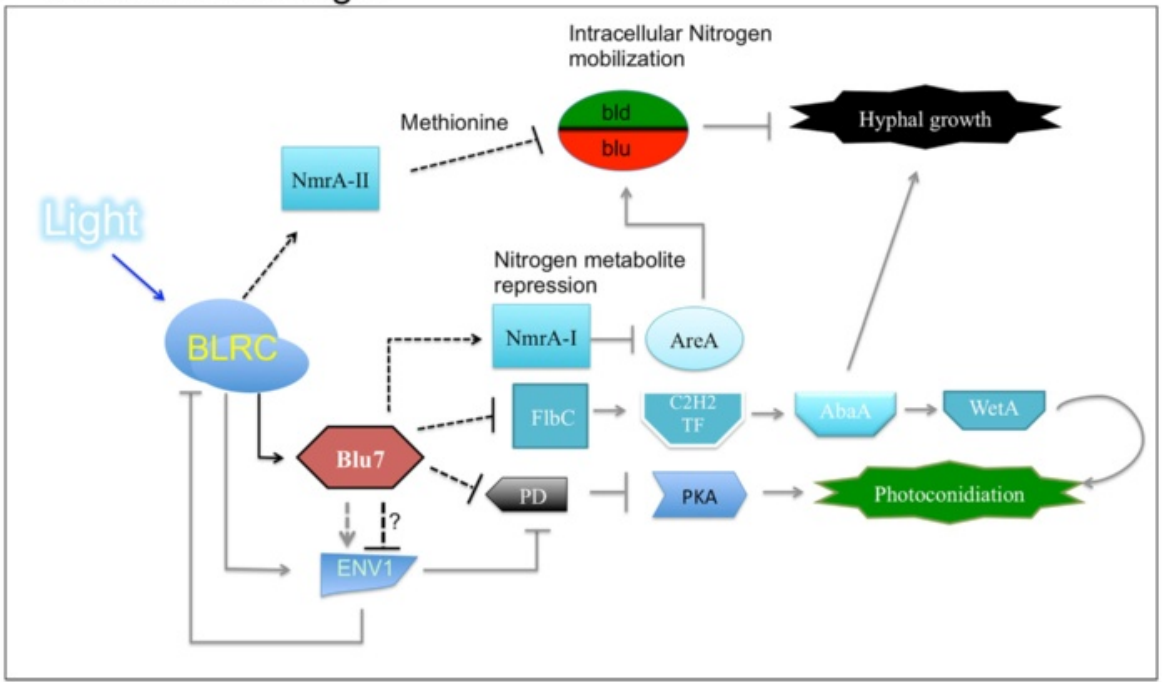

Fig. 9 Model of the role of the transcriptional regulator Blu7 in the molecular response of T. atroviride to light. a Molecular responses to a pulse of blue light. Exposure of $T$. atroviride to low fluence blue light perceived by the BLRC results in the transcriptional activation of blu7, sub1, azf1, $x / n r$ and 20 other TF encoding genes, as well as the induction of Envoy. Blu7 tunes photoconidiation by repressing the expression of the 5'-3' CAMP phosphodiesterase encoding gene (PD). Likewise, the induction of env1 enhances PD repression, while transitory accumulation of cAMP activates PKA that in turn regulates photoconidiation genes [34]. Nitrogen mobilization (amino acid transporters) is indirectly regulated by Blu7 and modulates the balance between conidiation and mycelial growth. b Molecular responses to continuous light exposure. Under constant white light, blu7 induction by the BLRC is regulated by the carbon source available in the medium. ENV1 negatively regulates BLRC target genes for adaptation to constant light. When T. atroviride is grown on glucose containing medium, blu7 limits the induction of env1. Blu7 is required for nitrogen mobilization through the inhibition of the orthologue of AreA by the negative regulator NmrA up-regulation. In addition, hyphal growth and conidiation are tightly regulated by the amount of FlbC to induce the sequential expression of the transcription factors $\mathrm{C} 2 \mathrm{H} 2 \rightarrow \mathrm{AbaA} \rightarrow$ WetA.

Black lines indicate control points supported by our RNA-seq data and gray lines are based on previously reported experimental evidence

$1.25 \mathrm{mM}, \mathrm{FeSO}_{4} 13.1 \mu \mathrm{M}, \mathrm{ZnSO}_{4} 7 \mu \mathrm{M}$ and $\mathrm{MnCl}_{2}$ $7.9 \mu \mathrm{M}$, and $2 \%$ glucose as carbon source, unless otherwise indicated, and $1.5 \%$ bacto-agar, and was adjusted to $\mathrm{pH}$ 4.8. Constant white light treatments were carried out with a fluorescent lamp at a $4.9 \mu$ molm $^{-2} \mathrm{~s}^{-1}$ fluence. Blue light treatments were carried out in a chamber equipped with light emitting diodes (LED). All other manipulations were carried out in the dark using only a safety red light with $0.5 \mu \mathrm{molm}^{-2} \mathrm{~s}^{-1}$ fluence.

\section{RNA and DNA manipulations}

The blu7 cDNA sequence was initially obtained from a standard cDNA library generated from polyA ${ }^{+}$RNA obtained from blue-light induced mycelia of $T$. atroviride. 
Since this cDNA appeared to be incomplete, we walked on the transcript using RT-PCR reactions and primers at different positions up-stream of the original transcript, aided by the genome sequence. The complete cDNA clone sequence was deposited in the GeneBank (Id KU666056). This sequence was supported by our Illumina transcriptome data (see below).

Gene replacement $\Delta b l u 7$ constructs were obtained by the double-joint PCR method [38]. The first PCR was made using the primers Pblu7-F, PQblu7-R and TQblu7F, Tblu7-R (Additional file 13) to amplify $1.4 \mathrm{~kb}$ fragments up- and down-stream of the 664 bp fragment to be replaced respectively, and joined them to $1.4 \mathrm{~kb}$ of the hygromycin phosphotransferase resistance cassette (hph), amplified by PCR from plasmid pCB1004 using the primers hph-F and hph-R (Additional file 13), in a second PCR without primers. Finally, the nested primers N5'-BLU7-F and N3'-BLU7-R were used in a third PCR reaction to amplify the $4.2 \mathrm{~kb}$ construct containing the joined 5'UTRblu7-hph-3'UTRblu7 fragments. Transformants overexpressing blu7 were obtained using a construct made by amplifying the cDNA of blu7 with the primers ORFBLU7-F and ORFBLU7-R and cloning it in TOPO-PCR 2.1. The plasmid was then digested with EcoRI and $B a m H I$, and the cDNA fragment inserted into EcoRI and BamHI sites of pUE10 [31]. The resulting plasmid carries the blu7 cDNA under the control of the constitutive promoter of the pyruvate kinase gene from T. reesei, and $1.4 \mathrm{~kb}$ of the 3' UTR of the blu17 gene of T. atroviride. Both constructions were directly used for PEG-mediated protoplast transformation of the WT strain as previously described [38]. After three rounds of single spore isolation, fungal DNA from the WT, $\Delta b l u 7$ and OEblu7 strains was obtained according to the protocol reported of Raeder and Broda [72]. Southern blot analysis of the $\Delta b l u 7$ mutants was carried out digesting genomic DNA with $\mathrm{XhoI}$, using as probe a $1.4 \mathrm{~kb}$ of the terminator region of blu7. For RNA preparation, mycelia were scraped from the surface of cellophane under red safelight $\left(0.05 \mu \mathrm{molm}^{-2} \mathrm{~s}^{-1}\right)$, immediately frozen in liquid nitrogen, and RNA extracted with TRIzol according to the manufacturer recommendations (Invitrogen, GIBCO-BLR).

\section{Light treatments of $\Delta b / u 7$ mutants}

Light-response curves of WT and $\Delta b l u 7$ mutants to blue light fluence were carried out in a growth chamber equipped with light-emitting diodes (Percival Scientific, Wisconsin, U.S.A.). A plug of mycelium from a colony grown on PDA for $48 \mathrm{~h}$ in darkness was used as inoculum in all experiments. Inocula of all strains (WT, $\Delta b l u 7$, OEblu7), for photoconidiation experiments, were cultivated in darkness for $36 \mathrm{~h}$ before exposure to a pulse of blue light of $50\left(2.9 \mu \mathrm{molm}^{-2} \mathrm{~s}^{-1}, 17.2 \mathrm{~s}\right), 80(2.9$ $\mu$ molm $\left.^{-2} \mathrm{~s}^{-1}, 27.6 \mathrm{~s}\right), 100\left(2.9 \mu \mathrm{molm}^{-2} \mathrm{~s}^{-1}, 34.5 \mathrm{~s}\right), 150$
(6.8 $\left.\mu \mathrm{molm}^{-2} \mathrm{~s}^{-1}, 22 \mathrm{~s}\right), 250\left(6.8 \mu \mathrm{molm}^{-2} \mathrm{~s}^{-1}, 30.7 \mathrm{~s}\right), 500$ (10.35 $\left.\mu \mathrm{molm}^{-2} \mathrm{~s}^{-1}, 34.5 \mathrm{~s}\right), 1200\left(28.2 \mu \mathrm{molm}^{-2} \mathrm{~s}^{-1}\right.$, $42.5 \mathrm{~s}), 2400$ (30.7 $\left.\mu \mathrm{molm}^{-2} \mathrm{~s}^{-1}, 78.2 \mathrm{~s}\right), 4800$ (30.7 $\left.\mu \mathrm{molm}^{-2} \mathrm{~s}^{-1}, 156.4 \mathrm{~s}\right), 9600\left(30.7 \mu \mathrm{molm}^{-2} \mathrm{~s}^{-1}, 312.7 \mathrm{~s}\right)$ and $11000\left(30.7 \mu \mathrm{molm}^{-2} \mathrm{~s}^{-1}, 358.3 \mathrm{~s}\right) \mu \mathrm{molm}^{-2}$, and then placed back in the dark for $36 \mathrm{~h}$. Conidia were collected from plates with a micropipette upon addition of $2 \mathrm{ml}$ sterile water to the plate and carefully scratching the surface of the colony with a sterile metal rod. Conidia from three independent biological replicates for each strain were then quantified using a hematocytometer.

For gene expression analysis of the blu7, env1, sah1 and gpd genes after a pulse of light, T. atroviride WT or $\Delta$ blu 7 mutant strain were exposed to $100 \mu \mathrm{molm}^{-2}$ (2.9 $\mu \mathrm{molm}^{-2} \mathrm{~s}^{-1}, 34.5 \mathrm{~s}$ ) of blue light using three biological replicates. After the blue light pulse, the mycelia were incubated in the dark for 30 min and collected for RNA extraction. Cultures of the WT or $\Delta b l u 7$ strains maintained always in the dark were used as controls of gene expression. Semiquantitative RT-PCR analyses were performed with superscript II transcriptase and Recombinant polymerase kits from Invitrogen. PCR's were carried out in a final volume of $25 \mu \mathrm{l}$ with the protocol specified by the manufacturer. To obtain the cDNA, $2 \mu \mathrm{g}$ of total RNA were used from each condition using reverse specific primers for each gene evaluated. The pairs of primers (Additional file 13) used were qBlu7-F - qBlu7-R for blu7, SAH1RT-5 F - SAH1RT-3R for sah1, ENVF - ENV-R for env1 and GPD-F - GDP-R for $g p d$. The PCR condition was $95{ }^{\circ} \mathrm{C}, 3 \mathrm{~min} ; 20$ cycles of $95{ }^{\circ} \mathrm{C}, 20 \mathrm{~s} ; 60{ }^{\circ} \mathrm{C}, 20 \mathrm{~s}$ and $72{ }^{\circ} \mathrm{C}, 20 \mathrm{~s}$; and a 1 min final extension at $72{ }^{\circ} \mathrm{C}$.

Growth assays under constant blue light of 4.9, 10 and $32 \mu \mathrm{molm}^{-2} \mathrm{~s}^{-1}$ were carried out using a light emitting diodes (LED) equipped chamber at $28{ }^{\circ} \mathrm{C}$ during $72 \mathrm{~h}$. Radial growth of the WT and $\Delta b l u 7$ mutant strains was determined every $12 \mathrm{~h}$.

To evaluate the impact of continuous exposure to light on growth with carbon limitation, the WT and $\Delta b l u 7$ mutant strains were cultivated in minimal media with $2 \%$ glucose, glycerol, mannitol or fructose under a 4.9 $\mu$ molm ${ }^{-2} \mathrm{~s}^{-1}$ white light at $28{ }^{\circ} \mathrm{C}$ during $72 \mathrm{~h}$.

Mycelium of the WT strain (IMI206040) and the $\Delta$ blu7 mutant collected after $60 \mathrm{~h}$ of growth under constant white light $\left(4.9 \mu \mathrm{molm}^{-2} \mathrm{~s}^{-1}\right)$ on glucose as sole carbon source was used for RNA-seq. Cultures of the WT and $\Delta b l u 7$ mutant maintained always in the dark during $60 \mathrm{~h}$ were used as controls for gene expression analyses. All experiments were carried out using three biological replicates for each strain in different days.

All RNA samples (Blue light pulse or continuous illumination) were processed and subjected to high-throughput sequencing using an Illumina $\mathrm{HiSeq}^{\mathrm{TN}} 2500$ in the core facilities of Cinvestav (Irapuato, Guanajuato, Mexico). 


\section{Analysis of the transcriptome data}

The Illumina sequencing data was grouped in 6 reads libraries of each light treatment for WT or $\Delta b l u 7$ mutant, 3 read libraries for the dark control and 3 for the light treatment, derived from three biological replicates carried out in independent days, starting from different pre-inoculum. Read libraries were mapped to the genome of T. atroviride using bowtie2 version 2.0.0beta7 with very sensitive default parameters [73, 74]. Reads counts were normalized by counts per million in the $\mathrm{R}$ version 2.15.2 environment using EDGER [75]. The same program was used to obtain differentially expressed genes using a cut-off of two-fold change and 0.01 of FDR between samples. Protein sequences in FASTA format was obtained from the Frozen gene catalog of T. atroviride genome V2 (http://genome.jgi.doe.gov/Triat2/Triat2.download.html). The corresponding proteins were compared against the MIPS database with a cutoff of $1 \times 10^{-3}$. GO term assignation was performed using an E-value $\leq 1 \times 10^{-3}$, an annotation score $\leq 40$, a GO weight of 5 [76]. Additionally, we used BLAST2GO with an E-value $<1 \times 10^{-3}$ for the annotation of all light regulated genes [77]. The differentially regulated genes associated proteins were annotated and showed as multilevel graphs by biological process, molecular function and cellular component, the representative level with more than 5 components was showed. GO enrichment analysis for each set of differentially expressed genes was performed using as reference the normalized set of genes with evidence of expression with at least two counts in one of the analyzed condition and with at least six counts among all conditions. In this analysis, Blast2GO [77] was used to compute enriched GO terms applying Fisher's exact test as implemented in GOSSIP [76, 78]. GO terms with 0.05 FDR and q-value $\leq 0.05$ were considered as significantly enriched in each comparison.

\section{Availability of data and materials}

RNA-Seq were deposited in the NCBI BioSample database (http://www.ncbi.nlm.nih.gov/biosample/) with the IDs SAMN04022924 and SAMN04025729, and linked to the sequence read archive (SRA, http://www.ncbi.nlm.nih.gov/sra) with the following IDs: SRS1050830 and SRS1051774. The sequence of the blu7 cDNA can be found in the GeneBank with accession number BankIt1891430 Seq1 KU666056.

\section{Additional files}

Additional file 1: Figure S1. A) Overview of $4500 \mathrm{bp}$ of the blu7 gene locus showing the reads mapping to this region. The JGl Genwise predicted structure for the blu7 gene (ID 284873) and that derived from our cDNA clone are schematically represented. B) Schematic representation of the domains found for the Blu7 deduced protein. The scheme indicates the proline rich region (PP, green box), the putative activation domain (QQQ, red box), the nuclear localization signal (NLS, light grey box) and the $\mathrm{C}_{2} \mathrm{H} 2$ zinc finger domain ( $\left.\mathrm{ZnF} \mathrm{C}_{2} \mathrm{H} 2\right)$. Numbers indicate amino acid positions. The dotted box shows the sequence deleted as a result of the gene replacement event. (JPG $1968 \mathrm{~kb}$ )

Additional file 2: Figure S2. Southern blot analysis of blu7 mutants. A) Schematic representation of the gene replacement event. Blue and red boxes represent the blu7 ORF and its 5' \& $3^{\prime}$ flanking regions, respectively; and restriction sites for Xhol, the enzyme used for DNA digestion indicated. The genes replacement construct is represented by the hygromycin resistance cassette (hph; green box), and the blu7 5' \& $3^{\prime}$ flanking regions (red boxes). The probe, a $1.4 \mathrm{~kb}$-long segment corresponding to the $3^{\prime}$ UTR of the blu7 locus, is also indicated. B) Autoradiogram of the Southern analysis. The expected $2.7 \mathrm{~kb}$ signal for the WT and the $4.6 \mathrm{~kb}$ for the $\Delta b / \mathrm{u} 7$ is shown due to a loss of the Xhol site by the $h p h$ replacement. (JPG $873 \mathrm{~kb}$ )

Additional file 3: Figure S3. Light growth response of the WT and $\Delta b / u 7$ strains under constant illumination. A) Growth rate of WT and $\triangle b l u 7$ mutant during $72 \mathrm{~h}$ in darkness (D) or constant white light. B) Growth rate of WT and $\Delta b / u 7$ mutant during $72 \mathrm{~h}$ in constant blue light. C) Growth rate of the $\triangle b / u 7$ mutant and WT under photoperiod of blue light- dark conditions during $72 \mathrm{~h}$. D) Conidia production of the WT and $\Delta b / u 7$ mutant under constant white $\left(3.2 \mu \mathrm{molm}^{-2} \mathrm{~s}^{-1}\right)$ or blue $\left(4.9 \mu \mathrm{molm}^{-2} \mathrm{~s}^{-1}\right)$ light treatments. One-way ANOVA and a pairwise $t$-test were applied to 6 independent replicates; asterisks indicate statistically significant differences $(a<0.05)$. (JPG $1758 \mathrm{~kb})$

Additional file 4: Table S1. List of responsive genes in WT or $\Delta b / u 7$ $30 \mathrm{~min}$ after a pulse of $100 \mathrm{\mu mol}$ of blue light compared to dark control of each strain. (CSV $77 \mathrm{~kb}$ )

Additional file 5: Figure S4. Heatmap of the overall light regulated genes of WT and $\Delta b / u 7$ mutant. Hierarchical clustering of the differential genes after a pulse of $100 \mu \mathrm{molm}^{-2}$ of blue light (A) or under constant white light (B) of the WT and $\triangle b / u 7$ mutant is shown. (JPG $1801 \mathrm{~kb}$ )

Additional file 6: Table S2. List of the most induced genes $30 \mathrm{~min}$ after a pulse of $100 \mathrm{mmolm}^{-2}$ of blue light shared in the mutant and the WT strain. (CSV $9 \mathrm{~kb}$ )

Additional file 7: Table S3. List of the most repressed genes after $30 \mathrm{~min}$ of $100 \mathrm{\mu molm}^{-2}$ of blue light shared in the mutant and the WT strain. (CSV $14 \mathrm{~kb}$ )

Additional file 8: Table S4. List of the most responsive genes present only in the WT or the $\triangle b l u 7$ mutant strains. (CSV 9 kb)

Additional file 9: Table S5. List of putative light responsive transcription factors. All putative transcription factors responding $30 \mathrm{~min}$ after a $100 \mu \mathrm{molm}^{-2}$ pulse of blue light in the WT or $\Delta b / u 7$ mutant are listed. (CSV $5 \mathrm{~kb}$ )

Additional file 10: Figure S4. A) Phenotype of the WT and $\Delta b / u 7$ after under constant blue light of $4.9,10$ and $32 \mathrm{mmolm}^{-2} \mathrm{~s}^{-1}$. B) Radial colony growth after $72 \mathrm{~h}$ of light treatment of the WT and $\Delta b / u 7$ strains. One-way ANOVA and a pairwise $t$-test were applied to data. Different letters indicate statistically significant differences $(a<0.05)$. (JPG $1062 \mathrm{~kb})$

Additional file 11: Figure S5. Stimulation of photoconidiation by several blue light fluences (from 50 to $11000 \mu \mathrm{molm}^{-2}$ ) in the WT, $\Delta b / u 7$ mutant and overexpressing (OEblu7) strains. (JPG $1487 \mathrm{~kb}$ )

Additional file 12: Table S6. List of genes responding after $60 \mathrm{~h}$ exposure to constant white light on glucose as carbon source compared to dark control in the WT or $\Delta b / u 7$ strains. (CSV $257 \mathrm{~kb}$ )

Additional file 13: Table S7. List of primers used in this study. Primers used to obtain the $\triangle b l u 7$ mutant and OEblu7 strains, as well as the primers used in RT-PCRs are listed. (CSV $1 \mathrm{~kb}$ )

\section{Abbreviations}

bld: blue light down-regulated; BLR: blue light regulator; blu: blue light up-regulated; CAMP: cyclic adenosine monophosphate; FC: fold change; FDR: false discovery rate; GO: gene ontology; PD: phosphodiesterase; RNA-seq: ribonucleic acid sequencing; TF: transcription factor; WC: white collar; WT: wild type. 


\section{Competing interests}

The authors declare that they have no competing interests.

\section{Authors' contributions}

Conceived and designed the experiments: JEC-Ch and AH-E Performed the experiments: JEC-Ch, EB-L and EUE-N Analyzed the data: JEC-Ch and AH-E Contributed analysis and wrote the paper: JEC-Ch and AH-E. All authors read and approved the final manuscript.

\section{Acknowledgements}

We wish to thank Dr. Miguel Hernández and M. Sc. Manuel Villalobos for assistance with the bioinformatics and statistical analyses. J.E. C-Ch. is indebted to CONACYT for a doctoral fellowship.

\section{Funding}

This work was supported by grant FORDECYT-2012-02-193512 from CONACYT to A H-E.

\section{Author details}

'Laboratorio Nacional de Genómica para la Biodiversidad, CINVESTAV Sede Irapuato, Km 9.6 Libramiento Norte Carretera Irapuato-León, 36821 Irapuato, Guanajuato, Mexico. ${ }^{2}$ Present Address: Unit for Basic and Applied Microbiology, Faculty of Natural Sciences, Autonomous University of Querétaro, Querétaro 76230, Mexico.

\section{Received: 19 August 2015 Accepted: 22 April 2016} Published online: 04 May 2016

\section{References}

1. Bahn Y, Xue C, Idnurm A, Rutherford JC, Heitman J, Cardenas ME. Sensing the environment: lessons from fungi. Nat Rev Microbiol. 2007;5:57-69.

2. Corrochano LM, Garre V. Photobiology in the Zygomycota: multiple photoreceptor genes for complex responses to light. Fungal Genet Biol. 2010;47:1-7.

3. Idnurm A, Verma S, Corrochano LM. A glimpse into the basis of vision in the kingdom Mycota. Fungal Genet Biol. 2010;47:881-92.

4. Rodriguez-Romero J, Hedtke M, Kastner C, Sylvia M, Fischer R. Fungi, hidden in soil or up in the air: light makes a difference. Annu Rev Microbiol. 2010; 64:585-610.

5. Hatakeyama R, Nakahama T, Higuchi Y, Kitamoto K. Light represses conidiation in Koji mold Aspergillus oryzae. Biosci Biotechnol Biochem. 2007;71:1844-9.

6. Blumenstein A, Vienken K, Tasler R, Purschwitz J, Veith D, FrankenbergDinkel N, Fischer R. The Aspergillus nidulans phytochrome FphA represses sexual development in red light. Curr Biol. 2005;15:1833-8.

7. Lee K, Singh P, Chung W, Ash J, Kim TS, Hang L, Park S. Light regulation of asexual development in the rice blast fungus, Magnaporthe oryzae. Fungal Genet Biol. 2006;43:694-706.

8. Herrera-Estrella A, Horwitz BA. Looking through the eyes of fungi: molecular genetics of photoreception. Mol Microbiol. 2007;64:5-15

9. Liu Y, He Q, Cheng P. Photoreception in Neurospora: a tale of two White Collar proteins. Cell Mol Life Sci. 2003;60:2131-8.

10. Schafmeier T, Diernfellner ACR. Light input and processing in the circadian clock of Neurospora. FEBS Lett. 2011;585:1467-73.

11. Harding RW, Turner RV. Photoregulation of the carotenoid biosynthetic pathway in albino and white collar mutants of Neurospora crassa. Plant Physiol. 1981;68:745-9.

12. Chen C-H, Dunlap JC, Loros JJ. Neurospora illuminates fungal photoreception. Fungal Genet Biol. 2010:47:922-9.

13. Froehlich AC, Noh B, Vierstra RD, Loros J, Dunlap JC. Genetic and molecular analysis of phytochromes from the filamentous fungus Neurospora crassa. Eukaryot Cell. 2005;4:2140-52.

14. Froehlich AC, Chen $\mathrm{C}-\mathrm{H}$, Belden WJ, Madeti C, Roenneberg T, Merrow M, Loros JJ, Dunlap JC. Genetic and molecular characterization of a cryptochrome from the filamentous fungus Neurospora crassa. Eukaryot Cell. 2010;9:738-50.

15. Chen C-H, Ringelberg CS, Gross RH, Dunlap JC, Loros JJ. Genome-wide analysis of light-inducible responses reveals hierarchical light signalling in Neurospora. EMBO J. 2009;28:1029-42.
16. Nsa IY, Karunarathna N, Liu X, Huang H, Boetteger B, Bell-Pedersen D. A novel cryptochrome-dependent oscillator in Neurospora crassa. Genetics. 2014;199:233-45.

17. Purschwitz J, Müller S, Kastner C, Schöser M, Haas H, Espeso EA, Atoui A Calvo AM, Fischer R. Functional and physical interaction of blue- and red-light sensors in Aspergillus nidulans. Curr Biol. 2008;18:255-9.

18. Bayram O, Braus GH, Fischer R, Rodriguez-Romero J. Spotlight on Aspergillus nidulans photosensory systems. Fungal Genet Biol. 2010;47:900-8.

19. Casas Flores S, Rios Momberg M, Bibbins M, Ponce Noyola P, Herrera Estrella A. BLR-1 and BLR-2, key regulatory elements of photoconidiation and mycelial growth in Trichoderma atroviride. Microbiology. 2004;150:3561-9.

20. Galun E, Gressel J. Morphogenesis in Trichoderma: Suppression of photoinduction by 5-Fluorouracil. Science. 1966;151:696-8.

21. Gressel J, Galun E. Morphogenesis in Trichoderma: Photoinduction and RNA. Dev Biol. 1967:15:575-98.

22. Rosales-Saavedra T, Esquivel-Naranjo EU, Casas-Flores S, Martínez-Hernández P, Ibarra-Laclette E, Cortes-Penagos C, Herrera-Estrella A. Novel light-regulated genes in Trichoderma atroviride: a dissection by cDNA microarrays. Microbiology. 2006;152:3305-17.

23. Wu C, Yang F, Smith KM, Peterson M, Dekhang R, Zhang Y, Zucker J, Bredeweg EL, Mallappa C, Zhou X, Lyubetskaya A, Townsend JP, Galagan JE, Freitag M, Dunlap JC, Bell-Pedersen D, Sachs MS. Genome-Wide characterization of light-regulated genes in Neurospora crassa. G3. 2014;4:1731-45.

24. Smith KM, Sancar G, Dekhang R, Sullivan CM, Li S, Tag AG, Sancar C, Bredeweg EL, Priest HD, McCormick RF, Thomas TL, Carrington JC, Stajich JE, Bell-Pedersen D, Brunner M, Freitag M. Transcription factors in light and circadian clock signaling networks revealed by genomewide mapping of direct targets for Neurospora White Collar Complex. Eukaryot Cell. 2010;9: 1549-56.

25. Colot HV, Park G, Turner GE, Ringelberg C, Crew CM, Litvinkova L, Weiss RL, Borkovich KA, Dunlap JC. A high-throughput gene knockout procedure for Neurospora reveals functions for multiple transcription factors. Proc Natl Acad Sci U S A. 2006;103:10352-7.

26. Ruger-Herreros C, Rodríguez-Romero J, Fernández-Barranco R, Olmedo M, Fischer R, Corrochano LM, Canovas D. Regulation of conidiation by light in Aspergillus nidulans. Genetics. 2011;188:809-22.

27. Adams TH, Wieser JK, Yu J-H. Asexual sporulation in Aspergillus nidulans. Microbiol Mol Biol Rev. 1998:62:35-54.

28. Boylan MT, Mirabito PM, Willett CE, Zimmerman CR, Timberlake WE. Isolation and physical characterization of three essential conidiation genes from Aspergillus nidulans. Mol Cell Biol. 1987;7:3113-8.

29. Marshall MA, Timberlake WE. Aspergillus nidulans WetA activates sporespecific gene expression. Mol Cell Biol. 1991;11:55-62.

30. Fuller KK, Ringelberg CS, Loros JJ, Dunlap JC. The fungal pathogen Aspergillus fumigatus regulates growth, metabolism, and stress resistance in response to light. MBio. 2013;4:e00142-13.

31. Esquivel-Naranjo EU, Herrera-Estrella A. Enhanced responsiveness and sensitivity to blue light by blr-2 overexpression in Trichoderma atroviride. Microbiology. 2007;153:3909-22.

32. Pall ML. Adenosine 3', 5'-phosphate in Fungi. Microbiol Rev. 1981;45:462-80.

33. Gerisch G. Cyclic AMP and other signals controlling cell development and differentiation in Dictyostelium. Annu Rev Biochem. 1987;56:853-79.

34. Casas-Flores S, Rios-Momberg M, Rosales-Saavedra T, Martínez-Hernández P, Olmedo-Monfil V, Herrera-Estrella A. Cross Talk between a fungal blue-light perception system and the cyclic AMP signaling pathway. Eukaryot Cell. 2006:5:499-506

35. Friedl MA, Kubicek CP, Druzhinina IS. Carbon source dependence and photostimulation of conidiation in Hypocrea atroviridis. Appl Environ Microbiol. 2008;74:245-50

36. Friedl MA, Schmoll M, Kubicek CP, Druzhinina IS. Photostimulation of Hypocrea atroviridis growth occurs due to a cross-talk of carbon metabolism, blue light receptors and response to oxidative stress. Microbiology. 2008;154:1229-41.

37. Tisch D, Kubicek CP, Schmoll M. New insights into the mechanism of light modulated signaling by heterotrimeric G-proteins: ENVOY acts on gna1 and gna3 and adjusts CAMP levels in Trichoderma reesei (Hypocrea jecorina). Fungal Genet Biol. 2011:48:631-40.

38. Yu J-H, Hamari Z, Han K-H, Seo J-A, Reyes-Domínguez Y, Scazzocchio C. Double-joint PCR : a PCR-based molecular tool for gene manipulations in filamentous fungi. Fungal Genet Biol. 2004;41:973-81. 
39. Paré A, Kim M, Juarez MT, Brody S, McGinnis W. The Functions of grainy head-like proteins in animals and fungi and the evolution of apical extracellular barriers. PLoS ONE. 2012;7, e36254.

40. Slattery MG, Liko D, Heideman W. The function and properties of the Azf1 transcriptional regulator change with growth conditions in Saccharomyces cerevisiae. Eukaryot Cell. 2006;5:313-20.

41. Newcomb LL, Hall DD, Heideman W. AZF1 is a glucose-dependent positive regulator of CLN3 transcription in Saccharomyces cerevisiae. Mol Cell Biol. 2002:22:1607-14.

42. Rothermel BA, Vega RB, Williams RS. The role of modulatory calcineurininteracting proteins in calcineurin signaling. Trends Cardiovasc Med. 2003;15-21.

43. Kingsbury TJ, Cunningham KW. A conserved family of calcineurin regulators. Genes Dev. 2000;14:1595-604.

44. Pinchai N, Perfect BZ, Juvvadi PR, Fortwendel JR, Cramer RA, Asfaw YG, Heitman J, Perfect JR, Steinbach WJ. Aspergillus fumigatus calcipressin CbpA is involved in hyphal growth and calcium homeostasis. Eukaryot Cell. 2009; 8:511-9.

45. Harren K, Schumacher J, Tudzynski B. The $\mathrm{Ca}^{2+} /$ Calcineurin-dependent signaling pathway in the gray mold Botrytis cinerea: the role of calcipressin in modulating calcineurin activity. PLoS ONE. 2012;7, e41761.

46. Tisch D, Schmoll M. Targets of light signalling in Trichoderma reesei. BMC Genomics. 2013;14:657.

47. Tisch D, Schmoll M. Light regulation of metabolic pathways in fungi. Appl Microbiol Biotechnol. 2009;85:1259-77.

48. Canessa P, Schumacher J, Hevia MA, Tudzynski P, Larrondo LF. Assessing the effects of light on differentiation and virulence of the plant pathogen Botrytis cinerea: characterization of the white collar complex. PLoS ONE. 2013;8, e84223.

49. Berlin $\mathrm{V}$, Yanofsky $C$. Isolation and characterization of genes differentially expressed during conidiation of Neurospora crassa. Mol Cell Biol. 1985;5: 849-55

50. Mikus M, Hatvani L, Neuhof T, Komoń-Zelazowska M, Dieckmann R, Schwecke T, Druzhinina IS, von Döhren H, Kubicek CP. Differential regulation and posttranslational processing of the class II Hydrophobin genes from the biocontrol fungus Hypocrea atroviridis. Appl Environ Microbiol. 2009:75:3222-9.

51. Nemcovic M, Farkas $V$. Stimulation of conidiation by derivatives of CAMP in Trichoderma viride. Folia Microbiol (Praha). 1998:43:399-402.

52. Scott WA, Solomon B. Adenosine 3',5'-cyclic monophosphate and morphology in Neurospora crassa: drug-induced alterations. J Bacteriol. 1975;122:454-63.

53. Berrocal-Tito GM, Rosales-Saavedra T, Herrera-Estrella A, Horwitz BA. Characterization of blue-light and developmental regulation of the photolyase gene phr1 in Trichoderma harzianum. Photochem Photobiol. 2000;71:662-8

54. Fuller KK, Loros JJ, Dunlap JC. Fungal photobiology: visible light as a signal for stress, space and time. Curr Genet. 2015;61:275-88.

55. Gessler NN, Aver'yanov AA, Belozerskaya TA. Reactive oxygen species in regulation of fungal development. Biochem Mosc. 2007;72:1091-109.

56. Belozerskaya TA, Gessler NN, Isakova EP, Deryabina YI. Neurospora crassa light signal transduction is affected by ROS. J Signal Transduct. 2012;2012:1-13.

57. Belozerskaya TA, Gessler NN. Oxidative stress and differentiation in Neurospora crassa. Microbiology. 2006;75:497-501.

58. Yoshida Y, Hasunuma K. Reactive oxygen species affect photomorphogenesis in Neurospora crassa. J Biol Chem. 2004;279:6986-93.

59. Schumacher J, Simon A, Cohrs KC, Viaud M, Tudzynski P. The transcription factor BCLTF1 regulates virulence and light responses in the necrotrophic plant pathogen Botrytis cinerea. PLoS Genet. 2014;10, e1004040. doi:10.1371/ journal.pgen.1004040.

60. Ballario P, Vittorioso P, Magrelli A, Talora C, Cabibbo A, Macino G. White collar-1, a central regulator of blue light responses in Neurospora, is a zinc finger protein. EMBO J. 1996;15:1650-7.

61. Castellanos F, Schmoll M, Martínez P, Tisch D, Kubicek CP, Herrera-Estrella A, Esquivel-Naranjo EU. Crucial factors of the light perception machinery and their impact on growth and cellulase gene transcription in Trichoderma reesei. Fungal Genet Biol. 2010;47:468-76.

62. Beltran Hernández EB. Envoy en la Fotorrespuesta de Trichoderma atroviride, Dissertation Universidad Michoacana de San Nicolás de Hidalgo. Morelia, Mexico: 2013.

63. Wilson RA, Arst HN. Mutational analysis of AREA, a transcriptional activator mediating nitrogen metabolite repression in Aspergillus nidulans and a member of the "streetwise" GATA family of transcription factors. Microbiol Mol Biol Rev. 1998;62:586-96.

64. Andrianopoulos A, Kourambas S, Sharp JA, Davis MA, Hynes MJ. Characterization of the Aspergillus nidulans nmrA gene involved in nitrogen metabolite repression. J Bacteriol. 1998;180:1973-7.

65. Fernandez J, Wright JD, Hartline D, Quispe CF, Madayiputhiya N, Wilson RA. Principles of carbon catabolite repression in the rice blast fungus: Tps1, Nmr1-3, and a MATE-family pump regulate glucose metabolism during infection. PLoS Genet. 2012;8, e1002673.

66. Werner AK, Sparkes IA, Romeis T, Witte C. Identification, biochemical characterization, and subcellular localization of allantoate amidohydrolases from Arabidopsis and soybean. Plant Physiol. 2008;146:418-30.

67. Donofrio NM, Oh Y, Lundy R, Pan H, Brown DE, Jeong JS, Coughlan S, Mitchell TK, Dean RA. Global gene expression during nitrogen starvation in the rice blast fungus, Magnaporthe grisea. Fungal Genet Biol. 2006;43:605-17.

68. Irniger S, Bäumer M, Braus GH. Glucose and Ras activity influence the ubiquitin ligases APC/C and SCF in Saccharomyces cerevisiae. Genetics. 2000;154:1509-21.

69. Rolland F, Winderickx J, Thevelein JM. Glucose-sensing and -signalling mechanisms in yeast. FEMS Yeast Res. 2002;2:183-201.

70. Krystofova S, Borkovich KA. The predicted G-protein-Coupled Receptor GPR-1 is required for female sexual development in the multicellular fungus Neurospora crassa. Eukaryot Cell. 2006;5:1503-16.

71. Brunner K, Omann M, Pucher ME, Delic M, Lehner SM, Domnanich $\mathrm{P}$, Kratochwill K, Druzhinina I, Denk D, Zeilinger S. Trichoderma G protein-coupled receptors : functional characterisation of a CAMP receptor-like protein from Trichoderma atroviride. Curr Genet. 2008;54:283-99.

72. Reader U, Broda P. Rapid preparation of DNA from filamentous fungi. Lett Appl Microbiol. 1985;1:17-20.

73. Langmead B, Salzberg SL. Fast gapped-read alignment with Bowtie 2. Nature Meth. 2012:9:357-9.

74. Langmead B, Trapnell C, Pop M, Salzberg SL. Ultrafast and memory-efficient alignment of short DNA sequences to the human genome. Genome Biol. 2009;10:R25

75. Robinson MD, Mccarthy DJ, Smyth GK. edgeR: a Bioconductor package for differential expression analysis of digital gene expression data. Bioinformatics. 2010;26:139-40.

76. Blüthgen N, Brand K, Cajavec B, Swat M, Herzel H, Beule D. Biological profiling of gene groups utilizing Gene Ontology. Genome Informatics. 2005;16:106-15.

77. Conesa A, Götz S. Blast2GO: A comprehensive suite for functional analysis in plant genomics. Int J Plant Genomics. 2008;2008:619832.

78. Conesa A, Götz S, García-gómez JM, Terol J, Talón M, Robles M. Blast2GO: a universal tool for annotation, visualization and analysis in functional genomics research. Bioinformatics. 2005;21:3674-6.

\section{Submit your next manuscript to BioMed Central and we will help you at every step:}

- We accept pre-submission inquiries

- Our selector tool helps you to find the most relevant journal

- We provide round the clock customer support

- Convenient online submission

- Thorough peer review

- Inclusion in PubMed and all major indexing services

- Maximum visibility for your research

Submit your manuscript at www.biomedcentral.com/submit 